\title{
MELNIKOV TRANSFORMS, BERNOULLI BUNDLES, AND ALMOST PERIODIC PERTURBATIONS
}

\author{
By \\ Kenneth R. Meyer \\ and \\ George R. Sell
}

IMA Preprint Series \# 358

October 1987

INSTITUTE FOR MATHEMATICS AND ITS APPLICATIONS UNIVERSITY OF MINNESOTA

514 Vincent Hall

206 Church Street S.E.

Minneapolis, Minnesota 55455 

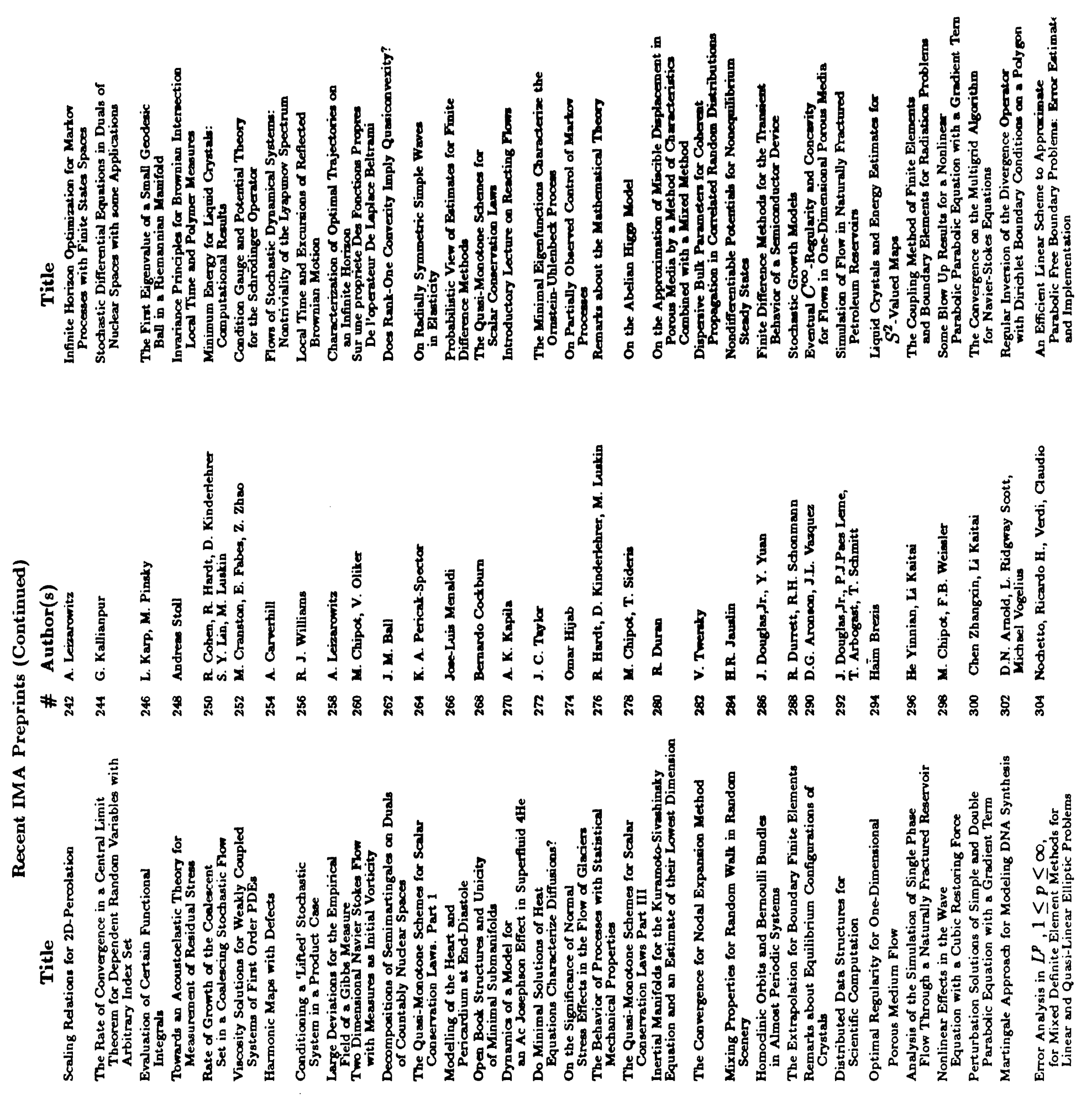

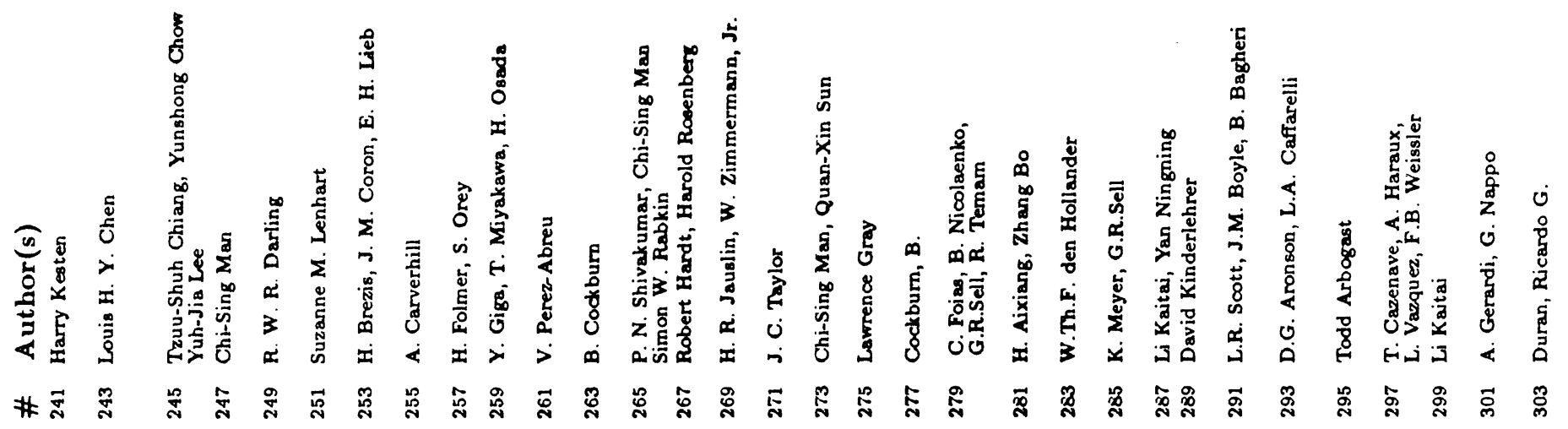




\title{
MELNIKOV TRANSFORMS, BERNOULLI BUNDLES, AND ALMOST PERIODIC PERTURBATIONS*
}

\author{
KENNETH R. MEYER $\dagger$ AND GEORGE R. SELL $\ddagger$
}

\begin{abstract}
In this paper we study nonlinear time-varying perturbations of an autonomous vector field in the plane $R^{2}$. We assume that the unperturbed equation, i.e. the given vector field has a homoclinic orbit and we present a generalization of the Melnikov method which allows us to show that the perturbed equation has a transversal homoclinic trajectory. The key to our generalization is the concept of the Melnikov transform, which is a linear transformation on the space of perturbation functions.

The appropriate dynamical setting for studying these perturbation is the concept of a skew product flow. The concept of transversality we require is best understood in this context. Under conditions whereby the perturbed equation admits a transversal homoclinic trajectory, we also study the dynamics of the perturbed vector field in the vicinity of this trajectory in the skew product flow. We show the dynamics near this trajectory can have the exotic behavior of the Bernoulli shift. The exact description of this dynamical phenomenon is in terms of a flow on a fiber bundle, which we call, the Bernoulli bundle.

We allow all perturbations which are bounded and uniformly continuous in time. Thus our theory includes the classical periodic perturbations studied by Melnikov, quasi periodic and almost periodic perturbations, as well as toroidal perturbations which are close to quasi periodic perturbations.
\end{abstract}

\section{INTRODUCTION}

In a seminal paper Smale (1963) introduced a very interesting geometric construction for showing that certain diffeomorphisms have infinitely many periodic points. The basic idea in this construction was to show that the existence of a certain "horseshoe" region implied that a full Bernoulli shift could be imbedded into the dynamics of the diffeomorphism. This horseshoe construction has since become an important technique in the study of dynamical systems, see for example Devaney (1986). While the horseshoe construction has many nice features, it is not without shortcomings. For example, in some problems the location of a horseshoe region is not always obvious, and sometimes requires extensive argumentation simply to show its existence.

More recently Palmer (1984) used the Lyapunov-Perron method (of integral equations) and the theory exponential dichotomies to develop an analytical approach, which when combined with the Melnikov method, has several very attractive features. It avoids completely the need for constructing a horseshoe region while at the same time gives a transparent and elementary proof of the imbedding of the Bernoulli shift flow. Also, as we shall see, it is the appropriate point of view for studying almost periodic perturbation of systems containing homoclinic orbits.

*This work was done in part at the Institute for Mathematics and its Applications and at the University of Cincinnati with funds made available by the National Science Foundation and the Applied and Computational Mathematics Program/DARPA.

†Department of Mathematical Sciences, University of Cincinnati, Cincinnati, Ohio 45221

$\ddagger$ Institute for Mathematics and its Applications, University of Minnesota, Minneapolis, Minnesota 55455 
By extending Palmer's ideas we present here a framework for generalizing the Bernoulli imbedding of Smale (1963) and the method of detecting transversal homoclinic orbits of Melnikov (1963) to almost periodic systems of differential equations. The key to this generalization is to use the Miller (1965) and Sell (1967) construction of a skew product flow over the hull of an almost periodic function. In this way the non-autonomous equations define a dynamical system whose structure reflects the geometry and spectra of the almost periodic forcing term. The main results of this paper where announced in Meyer and Sell (1987b).

Within the context of this skew product dynamical system we provide the appropriate generalizations of the following concepts: hyperbolic invariant sets, the stable and unstable manifolds, transversal homoclinic orbits, and shift automorphisms on a symbol space. This last object we call a Bernoulli bundle because it is a fiber bundle with fiber maps which are Bernoulli automorphisms. We then proceed to prove generalizations of (i) the Melnikov theorem for detecting homoclinic orbits, (ii) the shadowing lemma and (iii) Smale's theorem on the existence of a Bernoulli shift in the flow. One of the key ingredients for these generalizations is the Melnikov transform, which we introduce in this paper. In the context of almost periodic functions the Melnikov transform is a flow preserving mapping of the hull of one almost periodic function onto the hull of another. As we shall see, certain properties of the Melnikov transform will determine respective dynamical properties of the perturbed differential equation.

We first consider a basic model equation of the form

$$
\dot{x}=F(x)+\varepsilon f(t, x)
$$

where $x \in R^{2}, t \in R, \cdot=d / d t, F: R^{2} \rightarrow R^{2}$ is smooth and $f \in \mathcal{A P}$. Here $\mathcal{A P}$ is the collection of all functions $g: R \times R^{2} \rightarrow R^{2}$ where $g$ is almost periodic in $t$, uniformly for $x$, in compact subsets of $R^{2}$. The space $\mathcal{A P}$ has the compact open topology, i.e. the topology of uniform convergence on compact subsets of $R \times R^{2}$. We assume that the autonomous unperturbed system, when $\varepsilon=0$, has a non-degenerate saddle point $v_{0}$ and a homoclinic orbit (saddle connection) $u^{0}$ as pictured in Figure 1. Instead of just considering one function we take $f \in H \subset \mathcal{A P}$ where $H=H(g)$ is the hull of an almost periodic function $g$. Recall that if $g: R \times R^{2} \rightarrow R^{2}$ is almost periodic and $\tau \in R$, the $\tau$-translate of $g$ is $g_{\tau}(t, x)=g(t+\tau, x)$ and $H(g)$ is the closure, in the compact open topology, of the set of all translates of $g$.

The translational flow on $\mathcal{A P}$ is defined by

$$
\pi: R \times \mathcal{A P} \rightarrow \mathcal{A P}:(\tau, f) \rightarrow f_{\tau} .
$$

For any $g \in \mathcal{A P}$, the hull $H(g)$ is a compact invariant minimal set. When $g$ is quasi periodic $H$ turns out to be homeomorphic to a torus and this flow is equivalent to the standard irrational twist flow. When $g$ is limit periodic, $H$ is homeomorphic to a standard 


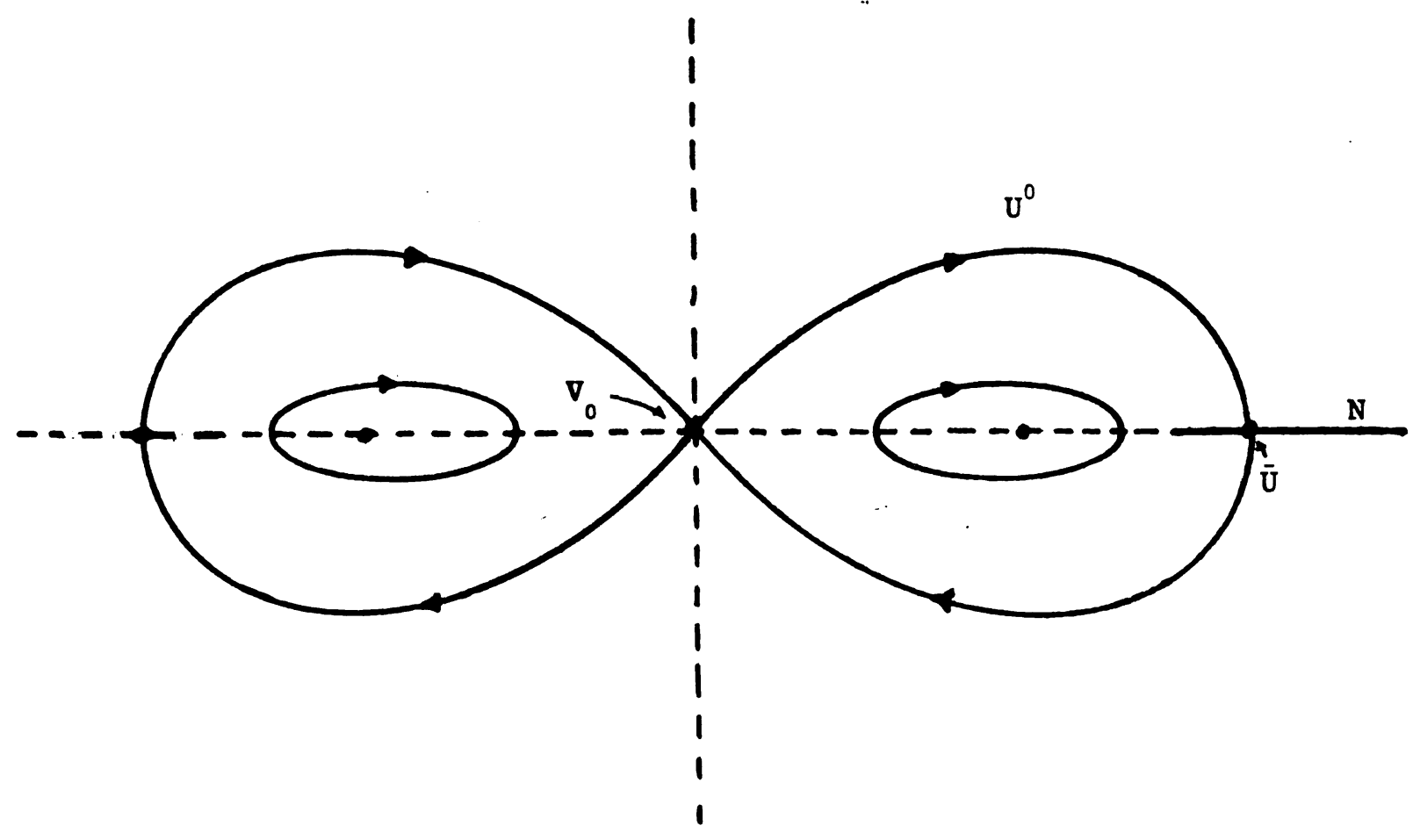

Figure 1: Duffing's Flow: The unperturbed phase portrait.

solenoid and the flow is equivalent to the supension of the classical adding machine map. Section 2 contains background material on almost periodic functions, the flow on their hull, cross sections to this flow and the general construction of the Bernoulli bundles. Two prototypical examples of a quasi periodic and a limit periodic function will be introduced in section 2 and used to illustrate our theory throughout this paper.

For $\varepsilon$ fixed we let $\phi\left(t, x_{0}, f\right)$ denote the solution of (1.1) which goes through $x_{0}$ at epoch $t=0$ and assume that $\phi$ is globally defined. Then the skew product flow defined by (1.1) is the flow

$$
\Pi: R \times\left(R^{2} \times H\right) \rightarrow R^{2} \times H:\left(t,\left(x_{0}, f\right)\right) \rightarrow\left(\phi\left(t, x_{0}, f\right), f_{t}\right) .
$$

Note the action on the second factor $\mathrm{H}$ is just the translational flow $\pi$, which we refer to as the base flow. Properties of this skew product flow are discussed in a greater detail in Section 2, however a few observations are useful at this point.

For $\varepsilon=0, \Pi$ decouples into a product flow and so the saddle point $v_{0}$ and the product of its stable and unstable manifolds with $H$ become important invariant sets for $\Pi$. Classical theorems on almost periodic differential equations establish the existence of similar 
invariant sets for $\Pi$ when $\varepsilon \neq 0$ but small. Of course these invariant sets for $\varepsilon \neq 0$ need not be product spaces, but they do reflect the dynamics for $\varepsilon=0$, see Sacker and Sell (1977) and Sell (1978). For example, the saddle point $\left\{v_{0}\right\} \times H$ becomes an invariant set for the skew product flow which is a 1-fold covering of $H$ and is flow equivalent to the translation flow on $H$ when $0<|\varepsilon| \ll 1$. These concepts and the generalization of transversal homoclinic orbit are given in Section 3. Interestingly, the Melnikov transform finds its natural domain of definition on the hull $H$, and its zero set in general defines a global cross section for the flow $\pi$ on $H$. Thus the Melnikov transform defines a cross section which in turn determines a time (the first return time), or a clock, which we use to describe the dynamics of the Bernoulli bundles. This clock is intimately connected with the spectrum of the almost periodic function $g$ and replaces the period in periodic systems. The main result of Section 3 is that if the Melnikov transform has a simple zero set then there exists a normally hyperbolic homoclinic bundle which, together with a suitable Shadowing Lemma, will beget the Bernoulli bundle.

Section 4 contains the generalization of hyperbolic invariant set for skew product flows and a simple proof of a generalization of the shadowing lemma based on the functional analytic proof given in Meyer and Sell (1987a). The shadowing lemma is the main tool we use to prove the existence of the Bernoulli bundle invariant set. For this purpose we follow Palmer (1984), who used the shadowing lemma to establish the existence of an invariant set in periodic systems which has a cross section map equivalent to the Bernoulli shift automorphism on a Cantor set. This idea avoids the problem of generalizing the geometric construction of the horseshoe region of Smale (1963). If the Melnikov transform has a simple zero set, then it is shown that $\Pi$ has a hyperbolic invariant set, which is simply the closure of this homoclinic set. The shadowing lemma then establishes the existence of orbits which are close to the homoclinic orbit for long periods but can take arbitrary small jumps from time to time. These theorems along with some examples are given in Section 5.

As an illustration of our theorems we show below that the perturbed Duffing's equation with negative linear stiffness

$$
u^{\prime \prime}-u+u^{3}=\varepsilon f(t)
$$

generates a Bernoulli bundle flow (along with the associated chaotic dynamics) for a very large class of perturbations $f$, including the quasi periodic example

$$
f(t)=a_{1} \cos \omega_{1} t+a_{2} \cos \omega_{2} t
$$

where $a_{1}, a_{2}$ are nonzero and $\omega_{1} / \omega_{2}$ is irrational.

Homoclinic orbits and their implications for autonomous and periodic systems have been investigated since their introduction by Poincare and therefore have a vast literature. The classics in the subject are Poincare (1892), Birkhoff (1932), Cartwright and Littlewood (1945), Melnikov (1963), and Smale (1963). Our work uses many of the ideas of Palmer 
(1984). The reader is referred to Chow and Hale (1982) and Guckenheimer and Holmes (1983) for a detailed discussion of and historical remarks on the autonomous and periodic literature.

Recently some related work has appeared on almost periodic systems. In Wiggens (1986b) the dynamical behavior of the Bernoulli bundle is described in the quasi periodic case. However, Wiggins (1968a) does not develop the connection with the Melnikov transform which we present here. Scheurle (1986) considers a system of almost periodic equations similar to ours, but considers only one equation and not a whole class of equations based on the hull of an almost periodic function. He uses the theory of exponential dichotomies, as extended by Palmer (1984), to find particular solutions which have a random structure. By not looking at the skew product flow defined by the equation he loses compactness and thus the uniformity needed to see the whole picture.

In another direction Ercolani, Forest and McLaughlin (1987) have shown that chaotic behavior can develop when one introduces arbitrarily small periodic forcing (with dissipation) to the sine-Gordon equation

$$
u_{t t}-u_{x x}+\sin u=0 .
$$

We believe that a straight forward extension of the theory we develop here can be used to explain this behavior. This problem is addressed in a sequel to this paper, Meyer and Sell (1987c).

While our primary interest is studying equation (1.1) when the perturbation term $f(t, x)$ is almost periodic in $t$, uniformly for $x$ in compact subsets of $R^{2}$, the theory we present here is has broader applications. By using the theory of skew product flows as developed in Sell (1967) and Sacker and Sell (1977) one can handle perturbations where $f(t, x)$ need not be almost periodic in $t$. For example, if $f(t, x)$ is uniformly continuous on sets of the form $R \times K$, where $K$ is compact in $R^{2}$, then the hull is still a compact invarient set. While we shall not do it here, one can also study perturbations $f(t, x)$ which are discontinuous in $t$, by using the generalizations as developed in Miller and Sell (1970), Artstein (1977) and Schwabik (1985). The key to all these considerations is the skew product dynamics which we present in Section 3.

We express our appreciaton to Clark Robinson who suggested the use of subshifts of finite type to describe our results.

\section{THE HULL, CROSS SECTIONS AND BERNOULLI BUNDLES}

Throughout this paper almost periodic will be in the sense of the founder of the subject, Bohr (1925ab, 1926, 1959); Besicovitch (1932) and others say that $f$ is uniformly almost periodic. Our function $f$ will depend on parameters, $x \in R^{n}$, and so the uniformity we speak of is with respect to these parameters. In this section we review some basic definitions and theorems so that we can develop the definition of the complicated flow 
on the spaces we call Bernoulli bundles. Basic references on almost periodic functions are Besicovitch (1932), Favard (1933), Bohr (1959), Corduneanu (1968), Sell (1971), Fink (1974), and Levitan and Zhikov (1982).

II.1 Almost Periodic Functions. A set $T \subset R=R^{1}$ is relatively dense if there is an $L>0$ such that any interval in $R$ of length $L$ contains a point of $T$. Let $f: R \times R^{n} \rightarrow R^{n}$ (or $R \times C^{n} \rightarrow C^{n}$ ) be continuous and let $K$ be a compact set in $R^{n}$ (or $C^{n}$ ). An $(\varepsilon, K$ )period for $f$ is a number $\tau$ such that $\|f(t+\tau, x)-f(t, x)\|<\varepsilon$ for all $(t, x) \in R \times K$. $f$ is almost periodic, uniformly for $x$ in compact sets, if it is bounded and for every $\varepsilon>0$ and every compact set $K$ in $R^{n}$ (or $C^{n}$ ), the set of $(\varepsilon, K)$-periodic for $f$ is relatively dense.

The spectral theory of an almost periodic function is based on the fact that the mean value

$$
M_{\omega}\{f\}(x)=\lim _{T \rightarrow \infty} \frac{1}{2 T} \int_{-T}^{T} f(s, x) e^{-i \omega s} d s
$$

exists, and for only a countable number of real numbers $\omega$ does one have $M_{\omega}\{f\} \neq 0$. The set $\left\{\omega: M_{\omega}\{f\} \neq 0\right\}$ is called the set of exponents or frequencies of $f$. We write

$$
f(t, x) \sim \sum_{-\infty}^{\infty} a_{k}(x) e^{i w_{k} t}
$$

where $a_{k}(x)=M_{\omega_{k}}\{f\}(x)$. This series is called the Fourier series of $f$. In general, the series given above does not converge to $f$ except in the mean square sense, i.e.,

$$
M\left\{\left\|f(t, x)-p_{N}(t, x)\right\|^{2}\right\} \rightarrow 0
$$

where $p_{N}(t, x)=\sum_{|k| \leq N} a_{k}(x) e^{i \omega_{k} t}$. Consider the real numbers $R$ as a vector space over the rational numbers $Q$. The smallest subspace $S \subset R$ which contains the set of exponents of $f$ is called the modulus of $f$.

In the case when $S$ is one dimensional, i.e. $\omega_{k}=r_{k} \alpha$ where $\alpha, r_{k} \in Q$, the function $f$ is said to be limit periodic, because in this case the partial sums $p_{N}$ of the Fourier series given above are periodic. Also, it can be shown that $f$ is limit periodic if and only if it is the uniform limit of periodic functions (not necessarly the partial sums $p_{N}$ ). The example we use throughout this paper is

$$
\ell(t)=\sum_{0}^{\infty} a_{k} e^{i 2 \pi\left(t / 2^{k}\right)}
$$

(or its real part) where the $a_{k}$ are constants chosen so that the series converges rapidly. The $N^{\text {th }}$ partial sum of $\ell$ is $2^{N}$-periodic.

If the modulus $S$ is a finite module over the integers, i.e., there exists a finite set $\lambda_{1}, \ldots, \lambda_{s} \in R$ such that $\omega_{k}=\sum a_{k i} \lambda_{i}$ with $a_{k i}$ integers, then $f$ is said to be quasi 
periodic. Equivalently, $f$ is quasi periodic if and only if there is a continuous function $F\left(\theta_{1}, \ldots, \theta_{s}\right)$, that is periodic in each argument, and real numbers $\lambda_{1}, \ldots, \lambda_{s}$ such that $f(t)=F\left(\lambda_{1} t, \ldots, \lambda_{s} t\right)$. Our standard example of a quasi periodic function will be

$$
q(t)=a_{1} e^{i \omega_{l} t}+a_{2} e^{i \omega_{2} t}
$$

(or its real part) where the ratio $\omega_{l} / \omega_{2}$ is irrational. Let $\mathrm{C}=\mathrm{C}\left(R \times R^{n}, R^{n}\right)$ (or $\mathrm{C}(R \times$ $C^{n}, C^{n}$ ) be the space of continuous functions from $R$ to $R^{n}$ (or $C^{n}$ ) with the topology of uniform convergence on compact sets (the compact open topology). Translations define a flow on $\mathcal{C}$ as follows

$$
\pi: R \times \mathfrak{C} \rightarrow \mathcal{C}:(\tau, f) \rightarrow f_{\tau}
$$

where $f_{\tau}(t, x)=f(t+\tau, x)$. For any $f \in C$ the orbit closure of $f$ is called the hull of $f$ and is denoted by $H(f)$. That is $H(f)=C l\left\{g_{\tau}: \tau \in R\right\}$, where the closure is taken in the compact open topology. Clearly $H(f)$ is invariant, but the hull of an almost periodic function is more. The following result can be found in Nemyckii and Stepanov (1960).

ThEOREM. If $f$ is almost periodic, then $H(f)$ is a compact minimal set. Furthermore, each element of $H(f)$ is almost periodic, the restriction $\pi \mid H(f)$ is equi-continuous, and $H(f)$ can be given a compact, connected, Abelian group structure.

The group structure + is defined as follows: First define $f_{\tau}+f_{\sigma}=f_{\tau+\sigma}$. Then if $f_{\tau_{n}} \rightarrow$ $a$ and $f_{s_{n}} \rightarrow b$ select a subsequence, if necessary, and define $a+b=\lim f_{t_{n}+s_{n}}$. It is not difficult to show that this is a well defined Abelian group operation. The mapping $\tau \rightarrow f_{\tau}$ is a homomorphism of $R$ onto a dense subgroup of $H(f)$ and so $H(f)$ is a compactification of $R$. One can also use the space $\mathcal{A P}$ of almost periodic functions with supremum norm. The above results hold in this space as well.

If $f$ is almost periodic with a Fourier series $f \sim \sum a_{k} e^{i \omega_{k} t}$ then the translate $f_{\tau}$ has the Fourier series $f_{\tau} \sim \sum a_{k} e^{i \omega_{k}(t+\tau)}$. For $g \in H(f)$ we obtain the Fourier series as follows: Let $f_{\tau_{n}} \rightarrow g$. By using the Cantor diagonalization procedure to select a subsequence, if necessary, we can assume that

$$
\tau_{n} \rightarrow \phi_{k} \quad \bmod 2 \pi / \omega_{k} \quad \text { for all } k \text {, as } n \rightarrow \infty \text {. }
$$

Then the Fourier coefficients of $f_{t_{n}}$ converge to the Fourier coefficients of $g$ or

$$
g \sim \sum a_{k} e^{i \omega_{k}\left(t+\phi_{k}\right)} .
$$

Thus if $g \in H(f)$ the phases $\phi_{k}$ are defined $\bmod 2 \pi / \omega_{k}$ such that (2.2) holds. As shown in the next paragraph, the converse is not true, i.e., one cannot choose $\phi_{k} \bmod 2 \pi / \omega_{k}$ arbitrarily and find a function $g \in H(f)$ such that (2.2) holds. 
Let $p(t)=e^{i t}+e^{i 2 t}$ so $p$ is $2 \pi$ periodic. It is easy to see that the hull of $p$ is precisely

$$
H(p)=\left\{e^{i(t+\alpha)}+e^{i 2(t+\alpha)}: 0 \leq \alpha<2 \pi\right\},
$$

which does not contain $e^{i t}+e^{i 2(t+1)}$ for example. It is easy to see that the map $\alpha \rightarrow$ $e^{i(t+\alpha)}+e^{i 2(t+\alpha)}$ is a topological homeomorphism and a continuous group isomorphism of $S=R /(2 \pi Z)$ onto $H(p)$. In general the hull of a periodic function is homeomorphic to a circle.

Let us now introduce the two prototypical examples which we will use to illustrate various aspects of our theory. The first is the quasi periodic function

$$
q(t)=a_{1} e^{i \omega_{1} t}+a_{2} e^{i \omega_{2} t}
$$

where $a_{1}, a_{2}$ are nonzero. We will be generally interested in the case where $\omega_{1} / \omega_{2}$ is irrational. The second example is the limit periodic function

$$
\ell(t)=\sum_{k=0}^{\infty} a_{k} e^{i 2 \pi\left(t / 2^{k}\right)}
$$

where the $a_{k}$ 's are nonzero and are chozen so that the series in (LP) converges uniformly and absolutely.

For (QP) assume that $\omega_{1} / \omega_{2}$ is irrational. By Kronecker's theorem, ${ }^{1}$ for any pair of real numbers $\alpha_{1}$ and $\alpha_{2}$ and any $\varepsilon>0$ there are integers $N_{1}$ and $N_{2}$ and a real number $\tau$ such that $\left|\tau \omega_{i}-2 \pi N_{i}-\alpha_{i}\right|<\varepsilon$. Consequently the translate $q_{\tau}$

$$
\begin{aligned}
q_{\tau}(t) & =a_{1} e^{i w_{1}(t+\tau)}+a_{2} e^{i w_{2}(t+\tau)} \\
& =a_{1} e^{i\left(w_{1} t+\alpha_{1}+\xi_{1}\right)}+a_{2} e^{i\left(w_{2} t+\alpha_{2}+\xi_{2}\right)}
\end{aligned}
$$

where $\left|\xi_{i}\right|<\varepsilon$. By taking $\varepsilon=1 / n$ and finding the corresponding $\tau=\tau_{n}$, one obtains

$$
q_{\tau_{n}}(t) \rightarrow a_{1} e^{i\left(\omega_{1} t+\alpha_{1}\right)}+a_{2} e^{i\left(\omega_{2} t+\alpha_{2}\right)}
$$

and so

$$
H(q)=\left\{a_{1} e^{i\left(\omega_{1} t+\alpha_{1}\right)}+a_{2} e^{i\left(\omega_{2} t+\alpha_{2}\right)}: \alpha_{i} \text { are defined } \bmod 2 \pi\right\} .
$$

By the uniqueness theorem for the Fourier series of an almost periodic function, distinct $\alpha_{i}$ 's $\bmod 2 \pi$ give rise to distinct elements in $H(q)$. Thus the map

$$
h: T^{2} \rightarrow H(q):\left(\alpha_{1}, \alpha_{2}\right) \rightarrow a_{1} e^{i\left(\omega_{1} t+\alpha_{1}\right)}+a_{2} e^{i\left(\omega_{2} t+a_{2}\right)}
$$

\footnotetext{
${ }^{1}$ See Fink (1974; page 58).
} 


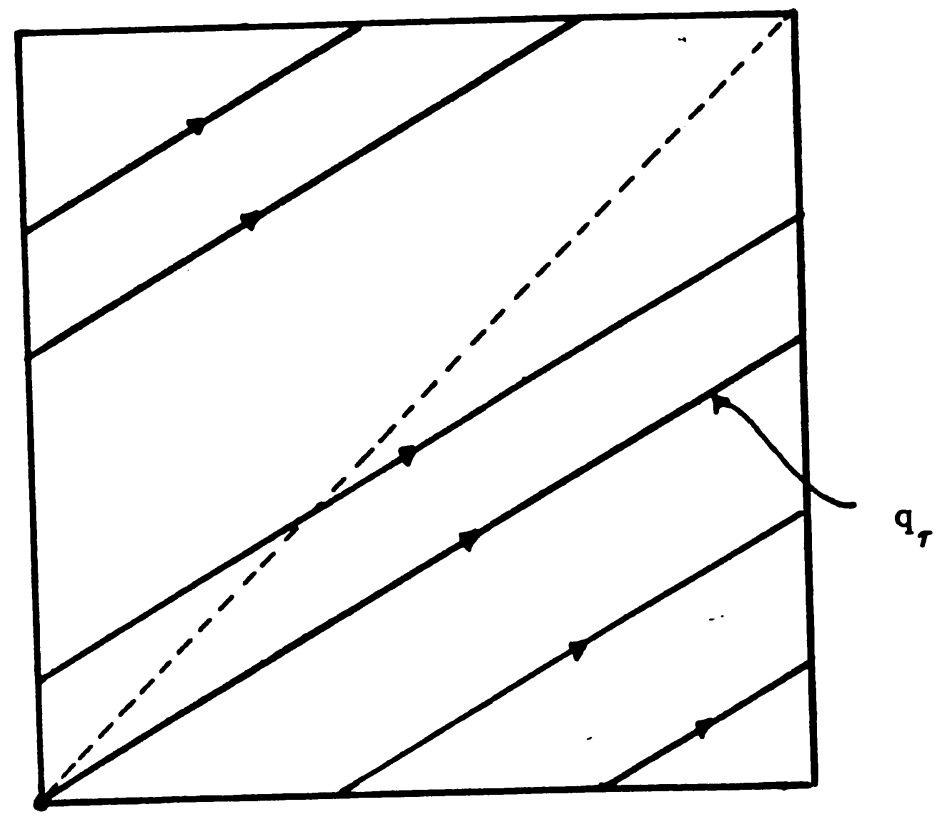

Figure 2: Quasi periodic hull: a torus.

is continuous, $1-1$ and onto and thus a homeomorphism of topological spaces. Also it carries the orbits of the dynamical system

$$
T^{2} \times R \rightarrow T^{2}:\left(\left(\alpha_{1}, \alpha_{2}\right), t\right) \rightarrow\left(\alpha_{1}+\omega_{1} t, \alpha_{2}+\omega_{2} t\right)
$$

onto the orbits of the translational flow and is an isomorphism of the topological groups. The function $q$ corresponds to $(0,0) \in T^{2}$ and the orbit of $q,\left\{q_{\tau}: \tau \in R\right\}$, corresponds to the dense line $\left\{\left(\omega_{1} t, \omega_{2} t\right): t \in R\right\}$ on $T^{2}$. See Figure 2. In general the hull of a quasi periodic function is an $s$-torus where $s$ is the dimension of the module $S$ over the integers $I$, see Pontryagin (1966).

An interesting extreme case related to the above occurs when the frequencies $\left\{\omega_{k}\right\}$ are independent over the rationals and not finite. Bohr (1959) calls this the disharmonic case, and he proves that the Fourier series converges uniformly to the function. The hull in this case is homoeomorphic to a countably infinite dimensional torus, i.e. a countable product of circles with the product topology.

Let us turn next to the limit periodic example $(L P)$. We shall give several characteri- 
zations of the hull $H(\ell)$. Let $\tau_{n}$ be a sequence such that $\ell_{\tau_{n}} \rightarrow \ell^{*}$ uniformly where

$$
\ell^{*}(t)=\sum_{0}^{\infty} a_{k} e^{i 2 \pi\left(t+\phi_{k}\right) / 2^{k}}
$$

We may assume that $\tau_{n} \rightarrow \phi_{k} \bmod 2^{k}$ as $n \rightarrow \infty$ and so $\tau_{n} \rightarrow \phi_{k+l} \bmod 2^{k+l}$, or

$$
\phi_{k}=\phi_{k+l} \bmod 2^{k}
$$

We claim that the hull of $\ell$ is precisely the set of functions $\ell^{*}$ as given in (2.3) where the $\phi_{k}$ are defined $\bmod 2^{k}$ and satisfy (2.4). Select representatives so that $0<\phi_{k}<2^{k}$, then condition (2.4) becomes $\phi_{k+l}=\phi_{k}+\sigma_{k} 2^{k}$ where $\sigma_{k}=0$ or 1 . Hence

$$
\phi_{N}=\phi_{0}+\sigma_{0}+\sigma_{1} 2^{1}+\cdots+\sigma_{N-1} 2^{N-1} \text {. }
$$

Let $\varepsilon>0$ be given and chose $N$ so large that $\sum_{N+1}^{\infty}\left|a_{k}\right|<\varepsilon$. Then the translate $\ell_{\phi_{N}}$ takes on the form

$$
\begin{aligned}
\ell_{\phi_{N}}(t) & =\sum_{k=0}^{N} a_{k} e^{i 2 \pi\left(t+\phi_{N}\right) / 2^{k}}+\xi_{N}(t) \\
& =\sum_{k=0}^{N} a_{k} e^{i 2 \pi\left(t+\phi_{k}\right) / 2^{k}}+\xi_{N}(t)
\end{aligned}
$$

where $\left|\xi_{N}(t)\right| \leq \varepsilon$. Thus $\ell_{\phi_{N}}(t) \rightarrow \ell^{*}(t)$ uniformly, consequently so the hull of $\ell$ is all $\ell^{*}$ of the form (2.3) with (2.4) holding.

By the uniqueness theorem for the Fourier series of an almost periodic function, distinct $\phi_{k}$ satisfying (2.4) give rise to distinct functions. Let $\tilde{\ell}$ denote another function in the hull of $\ell$ with phase angles $\left\{\tilde{\phi}_{k}\right\}$. Let $\varepsilon>0$ and $N$ be given and define

$$
U\left(\ell^{*}, N, \varepsilon\right)=\left\{\tilde{\ell} \in H(\ell):\left|\phi_{k}-\tilde{\phi}_{k}\right|<\varepsilon \text { for } k=l, \ldots, N\right\} .
$$

This set forms a base for the neighborhood system of $\ell^{*}$ in $H(\ell)$ since it is clear that if $N$ is large and $\varepsilon$ is small then $\ell^{*}$ and $\tilde{\ell}$ will be uniformly close. This suggests a coordinate system for $H(\ell)$ as an infinite product $\times_{k=0}^{\infty} S^{1}$ where $S^{1}=\left\{e^{i \theta}: 0 \leq \theta<2 \pi\right\}$ is the unit circle in the complex plane and the product has the usual product topology. We set $\theta_{k}=2 \pi \phi_{k} / 2^{k}$ so by $(2.4) \theta_{k}=2 \theta_{k+l}, \bmod 2 \pi$ and we set $z_{k}=e^{i \theta_{k}}$ so $z_{k}=z_{k+l}^{2}$. Then $\ell^{*}$ is given the coordinate $\left\{z_{0}, z_{1}, \ldots\right\} \in S$. Since $z_{k}=z_{k+l}^{2}$ the coordinates of $\ell^{*}$ are in the inverse limit system

$$
S_{2}: S^{1} \stackrel{z^{2}}{\leftarrow} S^{1} \stackrel{z^{2}}{\leftarrow} S^{1} \leftarrow \ldots
$$




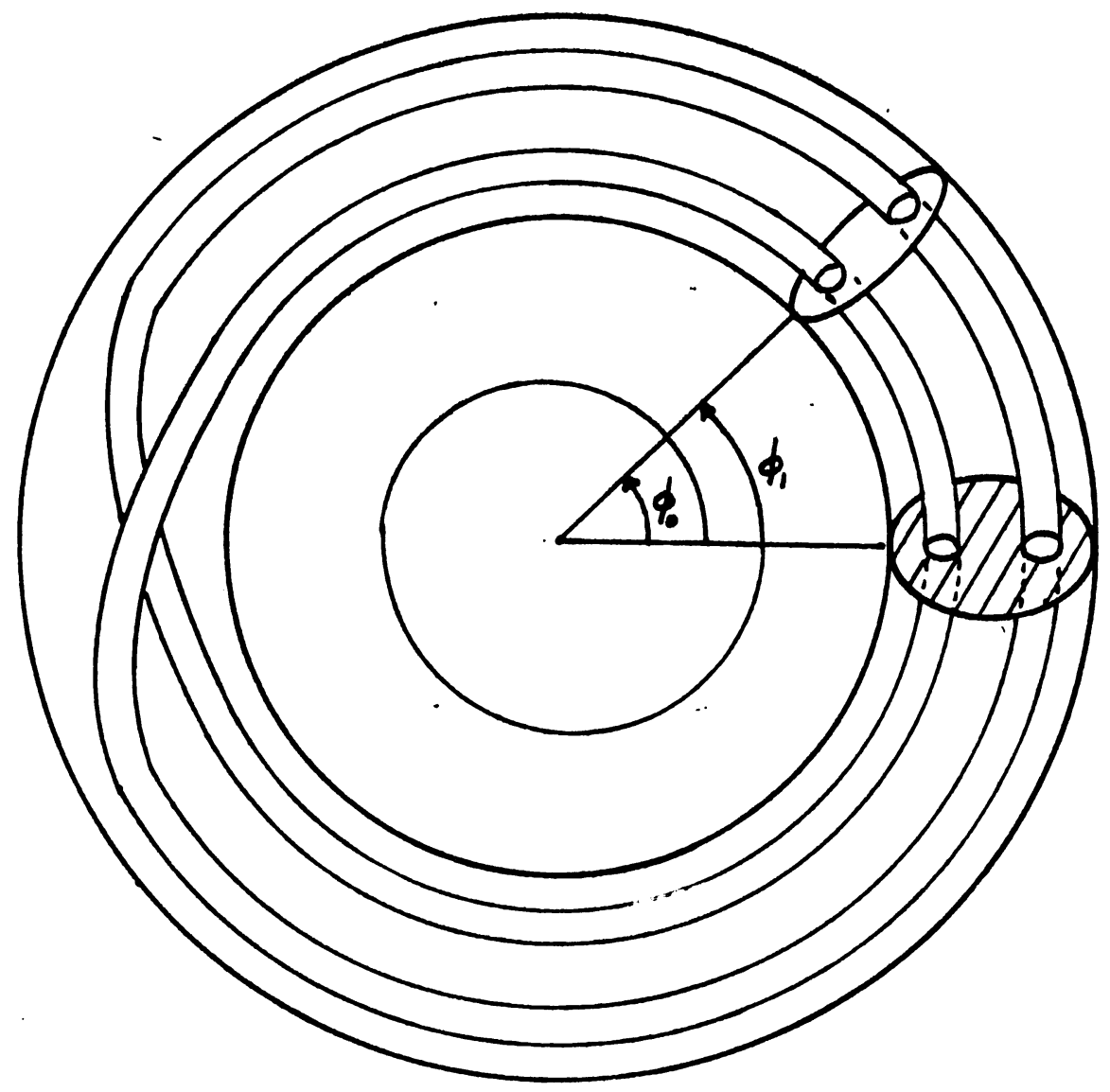

Figure 3: Limit periodic hull: a solenoid.

This is the classical coordinate system for the 2-solenoid as given in Hocking and Young (1961).

Let $T$ be a solid torus in a standard embedding in $R^{3}$ as given by rotating a meridianal disk $D(0)=\left\{(x, 0, z):(x-10)^{2}+z^{2} \leq 1\right\}$ about the $z$ axis as illustrated in Figure 3. (In Figure $3, D(0)$ is shaded.) Let $\phi_{0}$ be the polar angle in the $(x, y)$ plane normalized so that $\phi_{0}$ is defined mod 1 and let $D\left(\phi_{0}\right)$ be the image of $D(0)$ after being rotated by $\phi_{0}$. Let $T_{1}$ be a solid torus lying within the interior of $T_{0}$, longitudinally encircling $T_{0}$ twice and with medianal radius $1 / 4$ as illustrated in Figure 3. Let $\phi_{1}$ be an angular variable on $T_{1}$ which measures longitudinal displacement in $T_{1}$ and is defined mod 1 . As $\phi_{1}$ traverses $[0,2]$ the meridianal disk $D\left(\phi_{1}\right)$ in $T_{1}$ encircles the $z$-axis twice. Note that $T_{1}$ intersects $D\left(\phi_{0}\right)$ in two disks $D\left(\phi_{1}^{\prime}\right)$ and $D\left(\phi_{1}^{\prime \prime}\right)$ where $\phi_{0} \equiv \phi_{1}^{\prime} \equiv \phi_{1}^{\prime \prime} \bmod 1$.

Continue in this fashion to define $T_{k+1}$ encircling the torus $T_{k}$ twice with meridianal diameter $1 / 4^{k+1}$ with longitudinal angular coordinate $\phi_{k+1}$ defined $\bmod 2^{k+1}$. The 2solenoid $\sum_{2}$ is simply the intersection $\sum_{2}=\cap_{k=0}^{\infty} T_{k}$ which is a nonempty, connected, compact, one-dimensional subspace of $R^{3}$ and so is a "Klosed Kurve" in the sense of Menger. However, $\sum_{2}$ is not locally connected and hence cannot be a topological manifold. 
It is clear from the above construction that $H(\ell)$, the inverse limit system (2.5), and $\sum_{2}$ are all homeomorphic.

There is a standard minimal flow on $\sum_{2}$ which corresponds to the translation flow restricted to $H(\ell)$. The flow is defined by

$$
P_{t}\left(\ldots, z_{k}, \ldots\right)=\left(\ldots, z_{k} \exp \left(i 2 \pi t / 2^{k}\right), \ldots\right)
$$

which corresonds to uniform rotation about the $z$-axis in the solenoid $\sum_{2}$ as in Figure 3. The solenoid obtains a continuous Abelian group structure by component multiplication in the inverse limit representation, and this corresponds to the general Abelian group structure on the hull of any almost periodic function. The reader is referred to Nemytskii and Stepanov $(1960 ;$ p. 392) for a careful presentation of the minimal solenoid flow. These examples illustrate the general fact that compact Abelian groups are inverse limits of tori, see Pontryagin (1966).

The solenoid $\sum_{2}$ constructed above is the simplest of an uncountable number of solenoids which occur naturally in dynamical systems. This solenoid can be viewed as the Feigenbaum limit of a period doubling sequence. In the general construction of a solenoid the $T_{k+1}$ solid torus encircles the torus $T_{k}$ an arbitrary number of times, giving rise to uncountably many topologically distinct sets. Each of these solenoids carry a minimal flow, which Markus and Meyer (1980) show occur generically in Hamiltonian systems.

II.2 Cross Sections and Poincaré Mappings. Let $h: D \rightarrow D$ be any homeomorphism of a compact metric space $D$. Then $h$ defines a discrete dynamical system and let $T: D \rightarrow R$ be positive and continuous. The parallel flow $\gamma:(D \times R) \times R \rightarrow D \times R$ : $((d, \tau), t) \rightarrow(d, \tau+t)$ can be dropped to the quotient space $(D \times R) / \sim$ when $\sim$ is the equivalence relation $(d, t+T(d)) \sim(h(d), t)$. The flow on this quotient space is called the suspension of $h$, see Figure 4.

A flow $\pi: X \times R \rightarrow X$, where $X$ is a compact metric space, admits a (global) cross section $Z$ if i) $Z$ is a closed subset of $X$ ii) all trajectories meet $Z$ and iii) there is a positive continuous function $T: Z \rightarrow R$ such that $\pi(z, T(z)) \in Z$ and $\pi(z, t) \notin Z$ for all $z \in Z$ and $0<\tau<T(z)$. The function $T$ is called the first return time. The Poincaré map (or section map) is the map $\eta: Z \rightarrow Z: z \rightarrow \pi(z, T(z)$ ), which is a homeomorphism of $Z$ and defines a discrete dynamical system associated with the flow $\pi$. Flows that admit global cross sections are precisely supensions of discrete dynamical systems. See Ikegami (1969) and Neumann (1976) for more properties of cross sections.

A global cross section for a flow need not be connected. For example, if $\mathrm{X}$ is a nontrivial periodic orbit, then any nonempty finite subset of $\mathrm{X}$ is a global cross section.

The first return time $T: Z \rightarrow R$ is a continuous function defined on $Z$. By changing the time-scale, one can construct an equivalent flow $\hat{\Pi}$ where $T(z) \equiv 1$. While this fact is known, we present the basic ideas here. For $z \in Z$ and an integer $k$ define $\hat{\pi}(z, k)=\eta^{k}(z)$ 


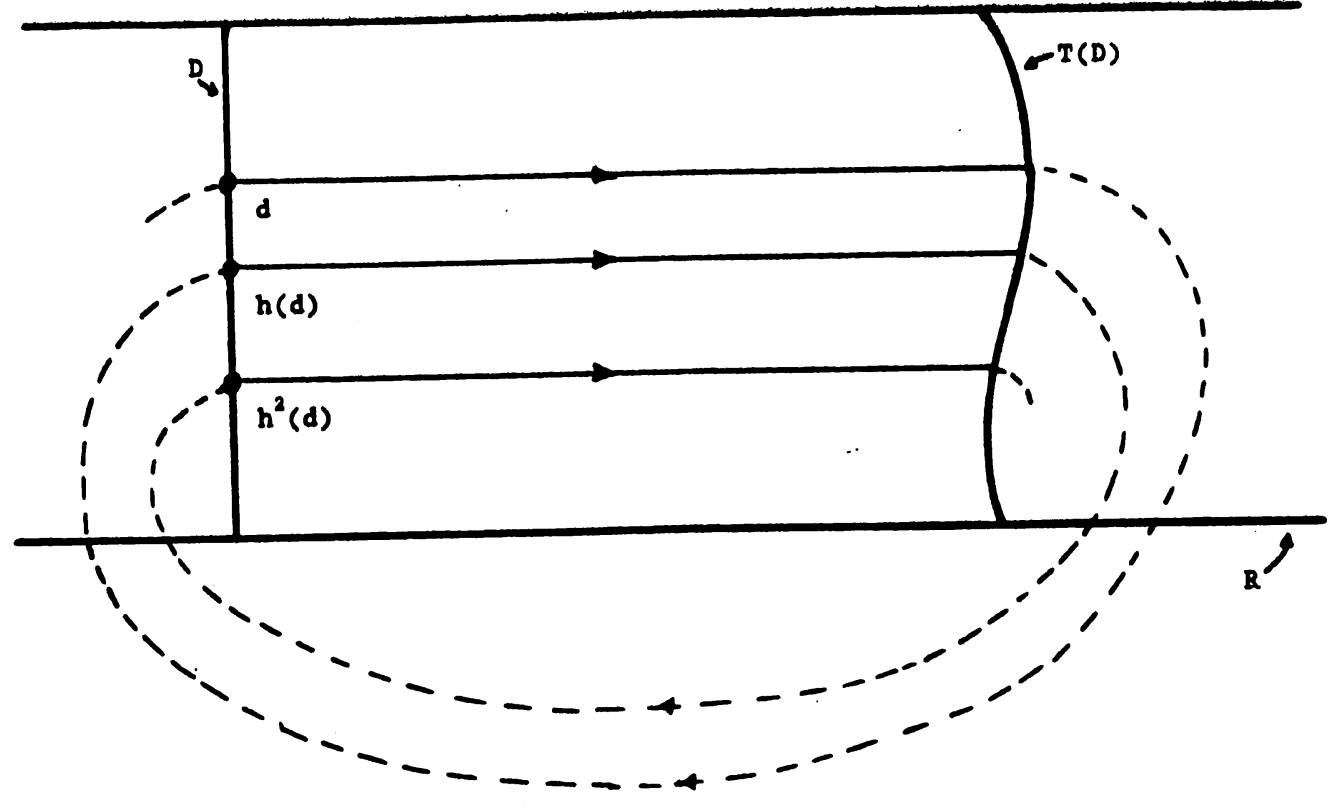

Figure 4. Suspension of a diffeomorphism $h$.

where $\eta$ is the Poincaré map. Next for $\tau \in R$ let $k$ be an integer with $\tau=k+\sigma$ where $\sigma \in[0,1)$ and define

$$
\hat{\pi}(z, \tau)=\pi\left(\eta^{k}(z), T\left(\eta^{k}(z)\right) \sigma\right), \quad z \in Z
$$

One can then show that $\hat{\pi}(z, \tau)$ is well-defined and that for any integer $\ell$ and any $\tau \in R$ one has

$$
\hat{\pi}(z, \ell+\tau)=\hat{\pi}(\hat{\pi}(z, \ell), \tau), \quad z \in Z
$$

Since $Z$ is a global cross section, it follows that for each $x \in X$, there is a unique $z_{x} \in Z$ and $t_{x} \in[0,1)$ with $x=\hat{\pi}\left(z, t_{x}\right)$. For $x \in X$ and $\sigma \in R$ one defines

$$
\hat{\pi}(x, \sigma)=\hat{\pi}\left(\hat{\pi}\left(z_{x}, t_{x}\right), \sigma\right)=\hat{\pi}\left(z_{x}, t_{x}+\sigma\right)
$$

It is not difficult now to show that $\hat{\pi}$ is a flow on $\mathrm{X}$ and that $Z$ is a cross section with first return time $\hat{T} \equiv 1$ and with Poincaré map $\eta$.

The translational flow on the hull of a nonconstant almost periodic function always admits a cross section. Let $\mathrm{f}$ be almost periodic and have a Fourier series as in (2.1). Then $g \in H(f)$ has a Fourier series (2.2) and

$$
g_{\tau}(t) \sim \sum a_{k} e^{i \omega_{k}\left(t+\phi_{k}+\tau\right)} \sim \sum a_{k} e^{i \omega_{k}\left(\phi_{k}+\tau\right)} e^{i \omega_{k} \tau}
$$

Thus the Fourier coefficient corresponding to the frequency $\omega_{k}$ is

$$
s_{k}\left(g_{\tau}\right)=M\left(g_{\tau}(t) e^{-i \omega_{k} t}\right)=a_{k} e^{i \omega_{k}\left(\phi_{k}+\tau\right)}
$$


which has a constantly changing argument as $\tau$ varies, provided $a_{k} \neq 0$ and $\omega_{k} \neq 0$. Since $g$ is nonconstant there is a $k$ with $a_{k} \neq 0$ and $\omega_{k} \neq 0$. We restrict now to such values $k$. Thus a cross section to this translational flow is given by

$$
Z=\left\{g \in H(f): \arg s_{k}(g)=0 \text { or } \phi_{k} \equiv 0 \bmod 2 \pi / \omega_{k}\right\} .
$$

In this case the first return time $T(z)=2 \pi / \omega_{k}$ is constant. Thus cross sections and their first return times are intimately connected with the Fourier spectrum of $g$.

Returning to the quasi periodic example $(Q P)$ we note that $q^{*} \in H(q)$ if and only if $q^{*}(t)=a_{1} e^{i\left(\omega_{1} t+\alpha_{l}\right)}+a_{2} e^{i\left(\omega_{2} t+\alpha_{2}\right)}$. Hence one cross section is $\alpha_{1}=0$ and the first return time is $2 \pi / \omega_{1}$. The angle $\alpha_{2}$ is a coordinate on $Z$, i.e. $Z$ is a circle in the torus. The Poincaré map in this coordinate system is $\eta: \alpha_{2} \rightarrow \alpha_{2}+\left(\omega_{2} / \omega_{1}\right) 2 \pi$ which is an irrational rotation of the circle.

For the limit periodic example $(L P)$, let $\ell^{*} \in H(\ell)$ be given by (2.3). As in the previous example we can define a cross section by requiring that the argument of one of the Fourier coefficients of $\ell^{*}$ be zero or equivalently that $\phi_{k} \equiv 0 \bmod 2_{k}$ for some fixed $k$. Consider the cross section $Z$ defined by $\phi_{0} \equiv 0 \bmod 1$ (the shaded disk in Figure 3), so the first return time is 1 . Clearly the intersection of this disk and $\sum_{2}$ is a Cantor set. However the associated Poincaré map is more easily understood in the coordinates given by the inverse limit system $S_{2}$ in (2.5). In that case $\left(z_{0}, z_{1}, \ldots\right) \in Z \subset S_{2}$ if and only if $z_{0}=1$. Thus

$$
z_{1} \in\{1,-1\}, \ldots, z_{k} \in e^{i j 2 \pi / 2^{k}}: \quad j=0,1, \ldots, 2^{k}-1 .
$$

The Poincaré map $\eta: Z \rightarrow Z$ is

$$
\eta:\left(\ldots, e^{i j 2 \pi / 2^{k}}, \ldots\right) \rightarrow\left(\ldots, e^{i(j+1) 2 \pi / 2^{k}}, \ldots\right) .
$$

In the $k$ th position the free index $j$ ranges over $0 \leq j<2^{k}$, which suggest using binary notation for $j$ with $k$ binary digits. That is in the $k^{\text {th }}$ position one has $j=b_{k} b_{k-l} \ldots b_{1}$. where $b_{j}=0$ or 1 . From (2.6) we see that the Poincaré map $\eta$ is just addition $\bmod 2^{k}$ in the $k^{\text {th }}$ position. Thus passing to the limit gives $Z=\times_{k=1}^{\infty} I_{2}$ with the usual product topology, where $I_{2}$ is a set consisting of two points and which is convenient to label as $I_{2}=\{1,2\}$. Then $z \in Z$ can be used as an infinite binary number $z=\ldots b_{3} b_{2} b_{1}$, where $\left(b_{j}+1\right) \in I_{2}$. The Poincaré map is just binary addition in this coordinate system and so is equivalent to the classical "adding machine" of dynamical systems. The adding machine is one of the simplest examples of a discrete almost periodic dynamical system.

II.3 Bernoulli Bundles. For $n \geq 2$, let $I_{n}=\{1,2, \ldots, n\}$ and $I_{\infty}=\{1,2, \ldots\}$. Define $B_{n}=\times_{k=-\infty}^{\infty} I_{n}$, i.e., $B_{n}$ is collection of all bi-infinite sequences on the symbols $s \in$ $I_{n}$. Thus if $q \in B_{n}$ then $q=\left(\ldots, q_{-1}, q_{0}, q_{1} \ldots\right)$ or more simply written $q=\ldots q_{-1} q_{0} . q_{1} \ldots$, where the zeroth position is to the left of the decimal point. Let $A: B_{n} \rightarrow B_{n}$ be the 
shift map or shift automorphism defined by $A(q)_{i}=q_{i+1}$, i.e., $A$ shifts the decimal point one place to the right. This is a classical dynamical system (see Morse (1921)) which has found many applications in contemporary dynamical systems, see for example Smale (1963) and Devaney (1986). The discrete dynamical system $\left(B_{n}, A\right)$ is sometimes referred to as the Bernoulli shift, or the full shift on $n$ symbols. For all finite values of $n$, $\left(B_{n}, A\right)$ is an invariant subset of $\left(B_{\infty}, A\right)$. There are other invariant subshifts which arise naturally in our theory, these are the subsets of finite type, which we define next.

A transition matrix is an $n \times n$ matrix $K=\left\{k_{i j}\right\}$ with entries which are either 0 or 1. For any transition matrix $K$ we define a subset $B_{n}(K)$ of $B_{n}$ by

$$
B_{n}(K)=\left\{q \in B_{n}: k_{q_{i} q_{i+1}}=1 \text { for all } i\right\} .
$$

In other words adjacent pairs of entries in a sequence $q \in B_{n}$ determine a location in the matrix $K$, the $q_{i} q_{i+1}$ position. Also the sequence $q$ is in $B_{n}(K)$ if and only if $k_{q_{i} q_{i+1}}=1$ for every $i$. Notice that $B_{n}(K)$ is $A$-invariant, i.e. $A B_{n}(K)=B_{n}(K)$. The set $B_{n}(K)$ is referred to as a subshift of finite type. Clearly $B_{n}(K)$ is a closed invariant subset of $B_{n}$.

The transition matrix $K$ serves as a lilany of which values $q_{j}$ may follow a given value $q_{i}$ for $q \in B_{n}(K)$ in the sense that one can have $q_{i+1}$ following $q_{i}$ if and only if $k_{q_{i} q_{i+1}}=1$, which we write as $q_{i} \rightarrow q_{i+1}$ for short. If one thinks of the digits $\{1,2, \ldots, n\}$ as representing states in a finite state automata and $K$ as being its transition matrix, then $q_{i} \rightarrow q_{i+1}$ illustrates the possible state jumps in $B_{n}(K)$. Alternatively the zeros in the transition matrix $K$ rules out certain adjacent pairs in a sequence $q \in B_{n}(K)$. For example if $K$ is a transition matrix where every entry is 1 , then $\left(B_{n}(K), A\right)$ is the full shift on $n$ symbols.

We will be primarily interested in a specific $n \times n$ matrix given by

$$
K=\left(\begin{array}{ccccccccc}
0 & 1 & 0 & 0 & 0 & \ldots & 0 & 0 & 0 \\
0 & 0 & 1 & 0 & 0 & \ldots & 0 & 0 & 0 \\
0 & 0 & 0 & 1 & 0 & \ldots & 0 & 0 & 0 \\
0 & 0 & 0 & 0 & 1 & \ldots & 0 & 0 & 0 \\
& & \vdots & & & \ddots & \vdots & & \\
0 & 0 & 0 & 0 & 0 & \ldots & 0 & 1 & 0 \\
0 & 0 & 0 & 0 & 0 & \ldots & 0 & 0 & 1 \\
1 & 0 & 0 & 0 & 0 & \ldots & 0 & 0 & 1
\end{array}\right)
$$

That is $K$ has 1's on the first super-diagonal and at the $(n, 1)$ and $(n, n)$ positions. Thus one has $1 \rightarrow 2,2 \rightarrow 3, \ldots, n-1 \rightarrow n, n \rightarrow n$, and $n \rightarrow 1$, see Figure 5 .

Let an overbar on a symbol, or on a sequence of symbols, mean that it is to be repeated infinitly often, so $\overline{1} .123 \overline{1}=\ldots 11.12311 \ldots$ The restriction of $A$ to $B_{n}(K)$, which we denote by $A$, has a unique fixed point $v^{*}=\bar{n} . \bar{n}$. Furthermore the point $u^{*}=\bar{n} .12345 \ldots n \bar{n}$ in 


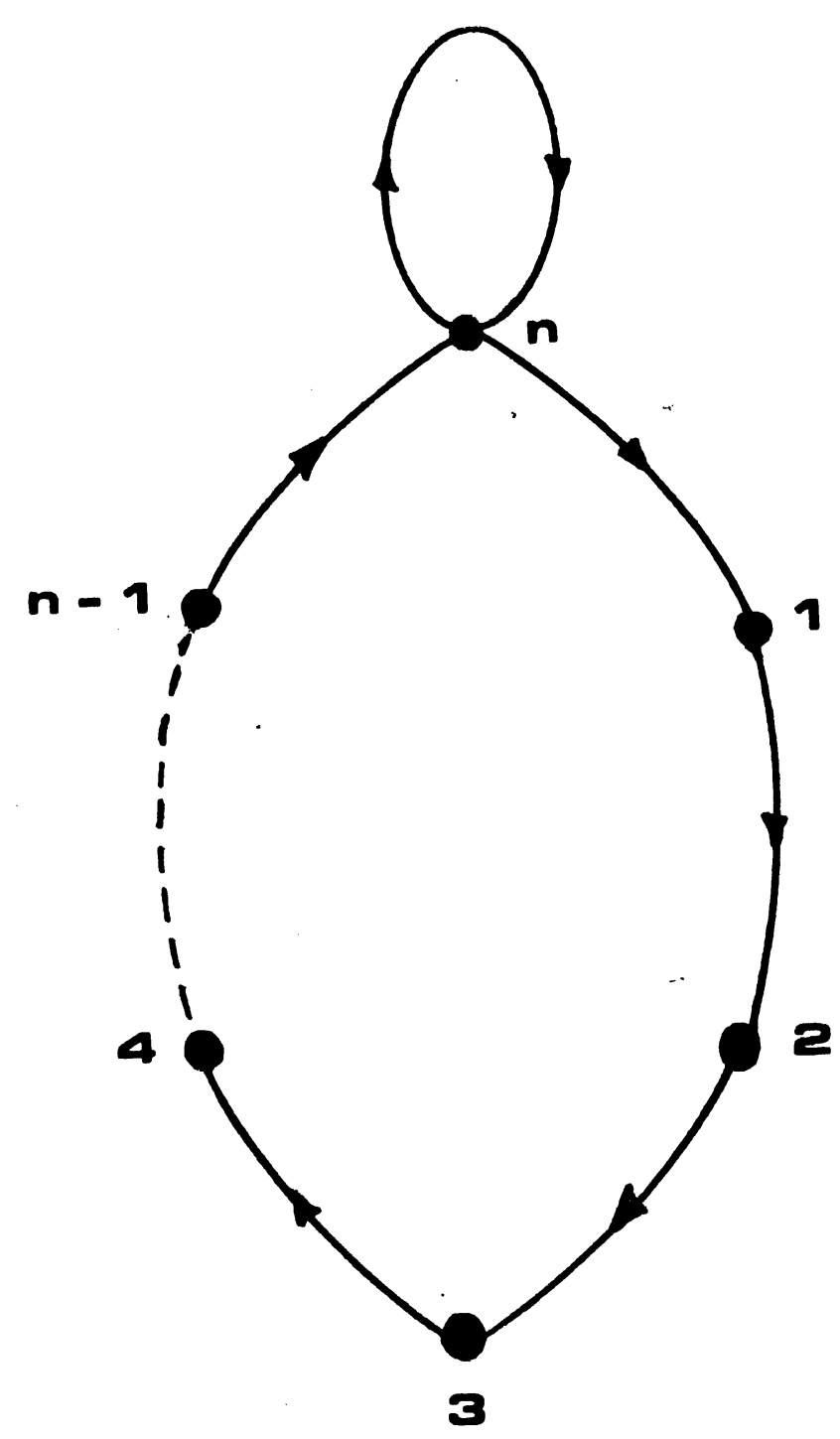

Figure 5: State transitions for $K$ given by (2.7).

$B_{n}(K)$ is doubly asymptotic, or homoclinic to $v^{*}$, i.e., $A^{t} u^{*} \rightarrow v^{*}$ as $|t| \rightarrow \infty$. For $p \leq n$ a point of period $p$ is given by $q=\overline{12 \ldots p} . \overline{12 \ldots p}$. Periodic points of period $p>n$ can be obtained by replacing the single $n$ in $\overline{12 \ldots n} . \overline{12 \ldots n}$ by a finite sequence of $(p+1-n) n$ 's. Thus $\left(B_{n}, A\right)$ contains periodic points of every period.

Since $B_{n}(K)$ is invariant under $A$, it is also invariant under various powers of $A$. One can readily compute the transition matrices for $A^{2}, A^{3}$, etc. For example if $K$ is given by (2.7) with $n=4$, then every entry in the transition matrix for $A^{6}$ is 1 . In other words, $\left(B_{4}(K), A^{6}\right)$ is a full shift on 4 symbols. More generally one can show that $\left(B_{n}(K), A^{2 n-2}\right)$ is a full shift on $n$ symbols when $K$ is given by (2.7).

The Bernoulli shift flow on $B_{n}$ is the prototypical example of chaotic behavior. Eventhough this is a completely deterministic dynamical event, it contains important features of randomness. The simplest way to see this is to take the view point of an observer who is able to watch a single coordinate of $q \in B_{n}$, say $q_{0}$, the entry to the left of the decimal point. After applying $A$ to $q$ the observer sees $q_{1}$. Since the observer does not know which $q \in B_{n}$ the shift mapping $A$ is operating on, the entry $q_{1}$ which appears after applying $A$ seems to be completely random. This form of randomness can be made more precise and 
we refer the reader to Sinai (1973), Alekseev (1976), Holmes (1980), Cornfeld, Fomin and Sinai (1982), and Marsden (1984) for further information.

Our next objective is to define a Bernoulli bundle $\mathcal{B}_{n}$ for $n \in\{2,3, \ldots, \infty\}$. This will be a fiber bundle over a space $X$ where the fibers are $B_{n}$. More precisely let $\pi$ be a flow on $\mathrm{X}$ with section $\mathrm{Z}$, and let $\eta: Z \rightarrow Z$ denote the Poincare map with first return time $T: Z \rightarrow R$. Let $K$ be any $(n \times n)$ transition matrix. The product of $A$ and $\eta$ is simply

$$
A \times \eta: B_{n}(K) \times Z \rightarrow B_{n}(K) \times Z:(q, z) \rightarrow(A(q), \eta(z)),
$$

The Bernoulli bundle $\mathcal{B}_{n}(K)$ is formed by projecting the parallel flow

$$
\begin{gathered}
\Psi:\left(B_{n}(K) \times Z \times R\right) \times R \rightarrow B_{n}(K) \times Z \times R \\
:((q, z, \tau), t) \rightarrow(q, z, \tau+t)
\end{gathered}
$$

onto the quotient space

$$
\mathcal{B}_{n}(K)=B_{n}(K) \times Z \times R / \sim
$$

where $(q, z, t+T(z)) \sim(A(q), \eta(z), t)$.

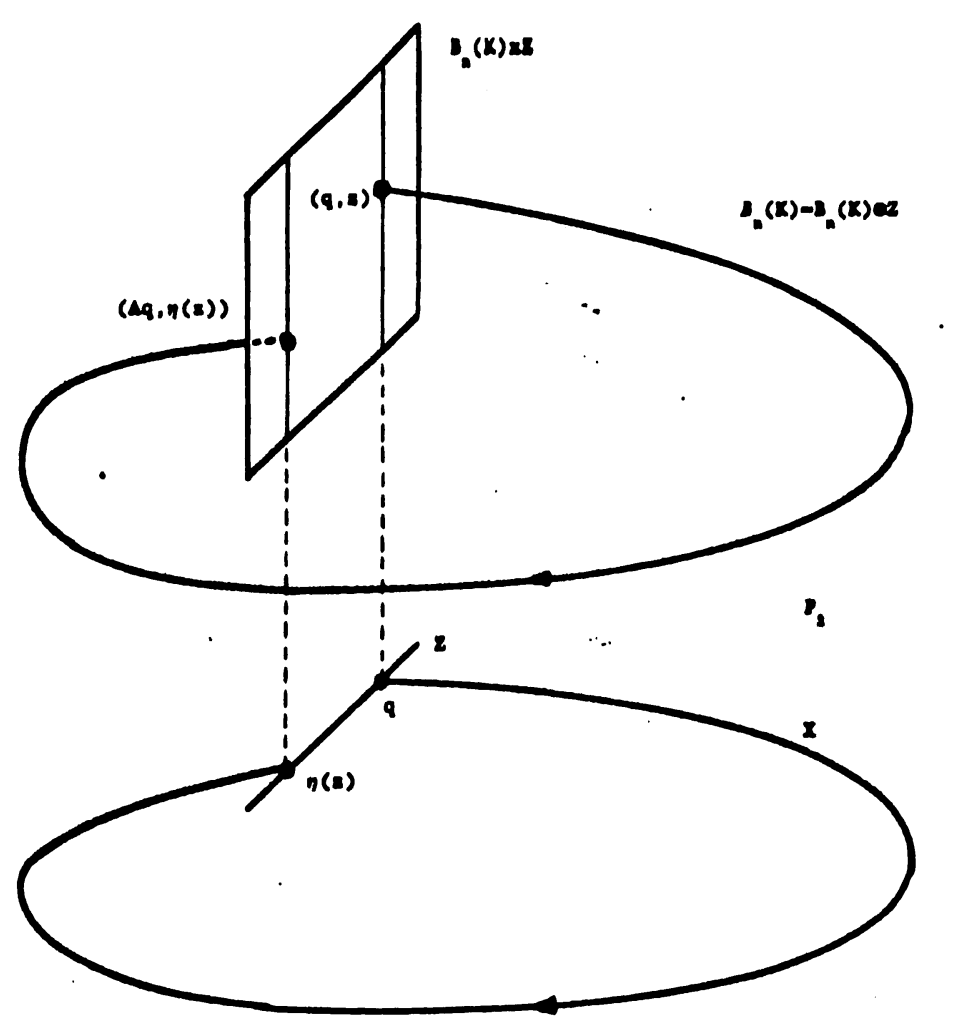

Figure 6: The Bernouli bundle flow. 
The flow on the Bernoulli bundle $\mathcal{B}_{n}(K)$ is given by (2.9) and we shall write

$$
\mathcal{B}_{n}(K)=B_{n}(K) \otimes X
$$

and denote the flow itself by $A \otimes \eta$. The notation in (2.10) emphasizes that $\left(\mathcal{B}_{n}(K), A \otimes \eta\right)$ is a skew product flow over $(X, \pi)$ and that the mapping

$$
P_{2}:(q, z, \tau) \rightarrow \pi(z, \tau)
$$

is a flow homomorphism, ${ }^{2}$ see Figure 6. Let $S \subset B_{n}(K)$ and $M \subset X$, and define

$$
S \otimes M=\left\{(s, m, \tau) \in \mathcal{B}_{n}(K): s \in S, m \in M, \tau \in R\right\} .
$$

Then $S \otimes M$ denotes the orbit in $\mathcal{B}_{n}(K)$ generated by $(S, M)$.

If $q_{0} \in B_{n}(K)$ is a fixed point of $A$, i.e., if $q_{0}=\bar{k} . \bar{k}$ for some $k \in I_{n}$, then $\left\{q_{0}\right\} \times X$ is a 1-cover of $X$. More generally if $q_{1} \in B_{n}(K)$ is a periodic point of $A$ of minimal period $p$, then $\left\{q_{1}\right\} \otimes X$ is a $p$-cover of $X$. If, in addition, $X$ is an almost periodic minimal set in the flow $\pi$, then $\left\{q_{1}\right\} \otimes X$ is an almost periodic minimal set in the flow $\Psi$ on $\mathcal{B}_{n}(K)$.

The Bernoulli shift space $B_{n}$ is metrizable. More specifically if $p, q \in B_{n}$ then

$$
d(p, q)=\sum_{m=-\infty}^{\infty} 2^{-|m|}\left|p_{m}-q_{m}\right|
$$

is a metric on $B_{n}$. The shift flow $A$ on $B_{n}$ is uniformly distal. Indeed if $p, q \in B_{n}$ with $p \neq q$ then there is an integer $k$ such that $d\left(A^{k} p, A^{k} q\right) \geq 1$. As a result the flow $A \otimes \eta$ on the Bernoulli bundle $\mathcal{B}_{n}(K)$ is distal whenever the flow $\pi$ on $X$ is distal.

One interesting application of the distality property occurs when $X$ is an almost periodic minimal set. In this case, if $M$ is a closed invariant set in $\mathcal{B}_{n}(K)$ with the property that for some $x \in X$ the fiber $P_{2}^{-1}(x)$ in $\mathcal{B}_{n}(K)$ is finite, then $M$ is the union of a finite number of almost periodic minimal sets, each of which is a finite covering space of $X$.

\section{The Melnikov Transform for Almost Periodic Systems}

III.1 The Induced Skew Product Flow. Consider the differential equation

$$
\dot{x}=F(x)+\varepsilon f(t, x)
$$

where $x \in R^{2}$, and $F: R^{2} \rightarrow R^{2}$ is smooth and $f \in \mathcal{G}$. Here $\mathcal{G}$ denotes the collection of all functions $f: R \times R^{2} \rightarrow R^{2}$ with the property that for every compact set $K \subset R^{2}$, (i) the function $f$ is uniformly continuous on $R \times K$ and (ii) there is a constant $k$ such that

$$
|f(t, x)-f(t, y)| \leq k|x-y|, \quad t \in R, x, y \in K .
$$

\footnotetext{
${ }^{2}$ Most of the conclusions in the remainder of this section follow from Sacker and Sell (1977).
} 
For many of our applications we will assume that $f \in \mathcal{A P}$, where $\mathcal{A P}$ consists of those functions from $\mathcal{G}$ which are almost periodic in $t$ uniformly for $x$ in compact sets in $R^{2}$.

On $\mathcal{G}$ we shall use the compact open topology, which means that a sequence $\left\{f_{n}\right\}$ converges to $f$ if and only if $f_{n} \rightarrow f$ uniformly compact subsets of $R \times R^{2}$. As before the translation of a function $f \in \mathcal{G}$ is given $f_{\tau}(t, x)=f(\tau+t, x)$ and the hull $H(f)$ is

$$
H(f)=C l\left\{f_{\tau}: \tau \in R\right\}
$$

One can show that if $f \in \mathcal{G}$ then $H(f)$ is a compact invariant set and $H(f) \subset \mathcal{G}$, cf. Sell (1967).

The unperturbed system when $\varepsilon=0$ is assumed to be Hamiltonian, and consequently, the trace of the Jacobian of $F$ is identically zero. (As a result the flow for the unperturbed equation is area-preserving in $R^{2}$.) Furthermore, the unperturbed system is assumed to have a non-degenerate saddle point at $v_{0} \in R^{2}$ and an orbit $u^{0}(t)$ homoclinic to $v_{0}$, i.e. $u^{0}(t) \rightarrow v_{0}$ as $t \rightarrow \pm \infty$. Thus the stable and unstable manifolds of $v_{0}$ intersect along the orbit of $u^{0}$. See Figure 1 .

Let $K$ be a large closed disk in $R^{2}$ which contains $v_{0}$ and the orbit $u^{0}(t)$ in its interior. For $\ell_{0}>0$ we define

$$
\mathcal{L}=\left\{f \in \mathcal{G}:|f(t, x)| \leq \ell_{0}, \text { for } t \in R, x \in K\right\}
$$

Let $0<\varepsilon_{0}$ be given. For $|\varepsilon| \leq \varepsilon_{0}$ we let $\phi\left(t, x_{0}, f, \varepsilon\right)$ be the solution of (3.1) that satisfies $\phi\left(0, x_{0}, f, \varepsilon\right)=x_{0}$. We consider $F$ as fixed and so suppress the dependence of the solution $\phi$ on $F$ for simplicity. However $f$ will be taken from a translation invariant compact subset $H \subset \mathcal{L}$, which oftentimes will be the hull of some almost periodic function.

In order to better understand the underlying geometry of (3.1) it is useful to introduce the concept of a skew product flow. Let $H$ be a translation invariant subset of $\mathcal{L}$. Assume, for simplicity, that the solution ${ }^{3} \phi\left(t, x_{0}, f, \varepsilon\right)$ of (3.1) is defined for all $t$ when $|\varepsilon|<\varepsilon_{0}$ and $f \in H$. The skew product flow on $R^{2} \times H$ is given by

$$
\Pi: R \times\left(R^{2} \times H\right) \rightarrow\left(R^{2} \times H\right):\left(t, x_{0}, f\right) \rightarrow\left(\phi\left(t, x_{0}, f, \varepsilon\right), f_{t}\right),
$$

see Miller (1965) and Sell (1967)

The next theorem, which is a classical result, describes how the hyperbolic fixed point $v_{0}$ behaves when $\varepsilon$ becomes nonzero.

\footnotetext{
${ }^{3}$ Since we shall only be interested in the behavior of solutions of (3.1) in the compact set $K$, there is no loss in generality in assuming the solutions of (3.1) to be defined for all $t \in R$. If this were not the case one could modify $F$ and $f$ near $x=\infty$, to accomplish this global existence property.
} 
THEOREM 0. There is an $\varepsilon_{0}, 0<\varepsilon_{0} \leq 1$, such that for all $f \in \mathcal{L},|\varepsilon|<\varepsilon_{0}$ there is a unique bounded solution $v(t, f, \varepsilon)$ of (3.1) that satisfies

$$
\sup _{f \in \mathcal{L}} \sup _{t \in R}\left|v(t, f, \varepsilon)-v_{0}\right| \rightarrow 0, \text { as } \varepsilon \rightarrow 0
$$

Furthermore, $v$ satisfies

$$
v\left(t, f_{\tau}, \varepsilon\right)=v(t+\tau, f, \varepsilon)=v_{\tau}(t, f, \varepsilon)
$$

If $f \in \mathcal{A P}$, then $v(t, f, \varepsilon)$ is also almost periodic in $t$, and the frequency module of $v$ is contained in the frequency module of $f$.

Proof. The proof of the existence of $v(t, f, \varepsilon)$ and the continuity in $\varepsilon$ follows from the usual infinite integral formulae involving exponential dichotomies, see Coppel (1965) or Hale (1969) for example. The proof of (3.3) is an immediate consequence of the uniqueness of $v$. Indeed both $v\left(t, f_{\tau}, \varepsilon\right)$ and $v(t+\tau, f, \varepsilon)$ are bounded solutions of the translated equation

$$
x^{\prime}=F(x)+\varepsilon f_{\tau}(t, x) \text {. }
$$

The conclusion on the almost periodic behavior follows from Sacker and Sell (1977).

With $\bar{x}(f, \varepsilon)=v(0, f, \varepsilon)$ one has $\phi(t, \bar{x}(f, \varepsilon), f, \varepsilon)=v(t, f, \varepsilon)$. Next define $V=\left\{(x(f, \varepsilon), f) \in R^{2} \times H: f \in H\right\}$, where $H$ is a translation invariant subset of $\mathcal{L}$. The identity (3.3) shows that $V$ is an invariant set for the flow $\Pi$ since

$$
\phi(t, \bar{x}(f, \varepsilon), f, \varepsilon)=v(t, f, \varepsilon)=v\left(0, f_{t}, \varepsilon\right)=\bar{x}\left(f_{t}, \varepsilon\right)
$$

The mapping $Q: H \rightarrow V: f \rightarrow(\bar{x}(f, \varepsilon), f)$ is a flow homomorphism by (3.3) also. In other words, the skew product flow defined by (3.1) has for small $\varepsilon$ an invariant set $V$ which is flow homomorphic to the translation flow on $H$.

III.2 The Stable and Unstable Bundles. Hale (1969) and Sell (1978) establish the local stable and unstable manifold theorem for (3.1). We will consider the global stable and unstable manifolds as well, since we are assuming the global existence of solutions of (3.1). The global version is obtained from the local version by the standard argument of backward and forward integration under the assumption that the solutions of (3.1) are defined for all $t$. See for example Palis and de Melo (1980) for a discussion of using backward integration to obtain the global stable manifold from the local stable manifold for autonomous equations. Since this argument has a straight forward extension to the skew product flows we consider here, we will apply it without further comment to our case.

Recall that the global stable manifold $W^{s}(f, \varepsilon)$ is defined as

$$
W^{s}(f, \varepsilon)=\left\{x_{0}:\left\|\phi\left(t, x_{0}, f, \varepsilon\right)-v(t, f, \varepsilon)\right\| \rightarrow 0 \text { as } t \rightarrow+\infty\right\}
$$


The local stable manifold theorem states that there is a sufficiently small $\delta>0$ such that

$$
W_{\mathrm{loc}}^{s}(f, \varepsilon) \stackrel{\text { def }}{=}\left\{x_{0} \in W^{s}(f, \varepsilon):\left\|\phi\left(t, x_{0}, f, \varepsilon\right)-v(t, f, \varepsilon)\right\|<\delta \text { for } t \geq 0\right\}
$$

is a smooth one-dimensional manifold. In particular, there is a smooth function

$$
w_{\mathrm{loc}}^{s}:(-\delta, \delta) \times H \times\left(-\varepsilon_{0}, \varepsilon_{0}\right) \rightarrow R^{2}
$$

such that

$$
W_{\mathrm{loc}}^{s}(f, \varepsilon)=\left\{w_{\mathrm{loc}}^{\mathrm{s}}(\sigma, f, \varepsilon): \sigma \in(-\delta, \delta)\right\}=\operatorname{Graph} w_{\mathrm{loc}}^{s}(\cdot, f, \varepsilon),
$$

and for fixed $(f, \varepsilon) \in H \times\left(-\varepsilon_{0}, \varepsilon_{0}\right)$ the map

$$
w_{\mathrm{loc}}^{\mathrm{s}}(\cdot, f, \varepsilon):(-\delta, \delta) \rightarrow R^{2}
$$

is an embedding of the interval $(-\delta, \delta)$ into $R^{2}$. The function $w_{l o c}^{s}$ is smooth in all its arguments. By backward integration $w_{\text {loc }}^{s}$ can be extended to

$$
w^{s}: R \times H \times\left(-\varepsilon_{0}, \varepsilon_{0}\right) \rightarrow R^{2}
$$

where the restriction $w \mid(-\delta, \delta) \times H \times\left(-\varepsilon_{0}, \varepsilon_{0}\right)=w_{\text {loc }}^{s}$ and so that the global stable manifold $W^{s}(f, \varepsilon)$ is the graph of $w^{s}$, i.e.

$$
W^{s}(f, \varepsilon)=\left\{w^{s}(\sigma, f, \varepsilon): \sigma \in R\right\},
$$

and for fixed $(f, \varepsilon)$ the map

$$
w^{s}(\cdot, f, \varepsilon): R \rightarrow R^{2}
$$

is an immersion of the line. For the skew product flow we define the stable bundle by

$$
\mathcal{W}^{s}(\varepsilon)=\left\{\left(W^{s}(f, \varepsilon), f\right): f \in H\right\}
$$

As was done in (3.3) one can establish that

$$
\phi\left(\tau, W^{s}(f, \varepsilon), f, \varepsilon\right)=W^{s}\left(f_{\tau}, \varepsilon\right),
$$

i.e. $\mathcal{W}^{s}(\varepsilon)$ is an invariant set for the skew product flow $\Pi$. Furthermore, $\mathcal{W}^{s}(\varepsilon)$ which is characterized by the formula

$$
\mathcal{W}^{s}(\varepsilon)=\left\{p \in R^{2} \times H: \Pi(t, p) \rightarrow V \text { as } t \rightarrow+\infty\right\} .
$$

$W_{\text {loc }}, W^{s}$ and $\mathcal{W}^{s}$ are called respectively the local stable manifold, the stable manifold and the stable bundle. By replacing $t \geq 0$ by $t \leq 0$ in (3.5) and $t \rightarrow+\infty$ by $t \rightarrow-\infty$ in (3.4) and (3.6) one defines the corresponding unstable sets $W_{\text {loc }}^{u}, W^{u}, \mathcal{W}^{u}$. 
Let $p=\left(x_{0}, f\right) \in \mathcal{W}^{s}(\varepsilon)$. We define the (partial) tangent space by

$$
T_{p} \mathcal{W}^{s}(\varepsilon)=\operatorname{span}\left\{\frac{\partial w^{s}}{\partial \sigma}\left(\sigma_{0}, f, \varepsilon\right)\right\}
$$

where $x_{0}=w^{s}\left(\sigma_{0}, f, \varepsilon\right)$. That is we consider only the component of the tangent space which lies in the plane $R^{2}$.

If $p \in \mathcal{W}^{s}(\varepsilon) \cap \mathcal{W}^{u}(\varepsilon)$ then we say $p$ is a homoclinic point (homoclinic to $V$ ) and $\{\Pi(t, p): t \in R\}$ is a homoclinic orbit. Thus $\Pi(t, p) \rightarrow V$ as $t \rightarrow \pm \infty$. If $p \in$ $\mathcal{W}^{s}(\varepsilon) \cap \mathcal{W}^{u}(\varepsilon)$ and $T_{p} R^{2}=R^{2}=T_{p} \mathcal{W}^{s}(\varepsilon)+T_{p} \mathcal{W}^{u}(\varepsilon)$, then we say $\mathcal{W}^{s}(\varepsilon)$ and $\mathcal{W}^{u}(\varepsilon)$ intersect transversally at $p$. If at each point $p \in \mathcal{W}^{s}(\varepsilon) \cap \mathcal{W}^{u}(\varepsilon), \mathcal{W}^{s}(\varepsilon)$ and $\mathcal{W}^{u}(\varepsilon)$ intersect transversally at $p$, then we say $\mathcal{W}^{s}(\varepsilon)$ and $\mathcal{W}^{u}(\varepsilon)$ intersect transversally and write $\mathcal{W}^{s}(\varepsilon) \bar{\AA} \mathcal{W}^{u}(\varepsilon)$.

III.3 The Melnikov Transform. The Melnikov functional gives a criteria for the existence of transversal homoclinic orbits. At a point $\bar{u}=u^{0}(0)$ on the homoclinic orbit of the unperturbed system we construct a normal line $n$ perpendicular to $F(\bar{u})$ and measure the separation of the stable and unstable manifolds on this normal, see Figure 1 and 7. We differ from the presentation in Melnikov (1963), Guckenheimer and Holmes (1983) and others by fixing the normal $n$ and measuring the separation at the epoch $t=0$. While this may appear to be restrictive, we will see that this is the correct approach for generalizing the Melnikov method to nonperiodic perturbations. It is important to note that we allow $f$ to vary over the compact invariant set $H$.

For $f \in H$ and $\varepsilon$ small there are unique solutions

$$
\begin{aligned}
& u_{s}(t, f, \varepsilon)=u^{0}(t)+\varepsilon u_{s}^{1}(t, f)+0\left(\varepsilon^{2}\right) \\
& u_{u}(t, f, \varepsilon)=u^{0}(t)+\varepsilon u_{u}^{1}(t, f)+0\left(\varepsilon^{2}\right)
\end{aligned}
$$

such that

$$
\begin{aligned}
& u_{s}(t, f, \varepsilon) \rightarrow v(t, f, \varepsilon) \text { as } t \rightarrow+\infty \\
& u_{u}(t, f, \varepsilon) \rightarrow v(t, f, \varepsilon) \text { as } t \rightarrow-\infty
\end{aligned}
$$

$u_{s}(0, f, \varepsilon) \in n$ and $u_{u}(0, f, \varepsilon) \in n$. Thus $u_{s}$ and $u_{u}$ are the unique solutions on the stable and unstable manifold which at $t=0$ lie on the normal $n$, cf. Figure 7 .

We define the separation of the stable and unstable manifold on $n$ to be

$$
d(f, \varepsilon)=\left\|u_{s}(0, f, \varepsilon)-u_{u}(0, f, \varepsilon)\right\|
$$

where $\|\cdot\|$ denotes the Euclidean norm on $R^{2}$. Equivalently one has

$$
d(f, \varepsilon)=\operatorname{dist}\left(W^{s}(f, \varepsilon) \cap n, W^{u}(f, \varepsilon) \cap n\right)
$$




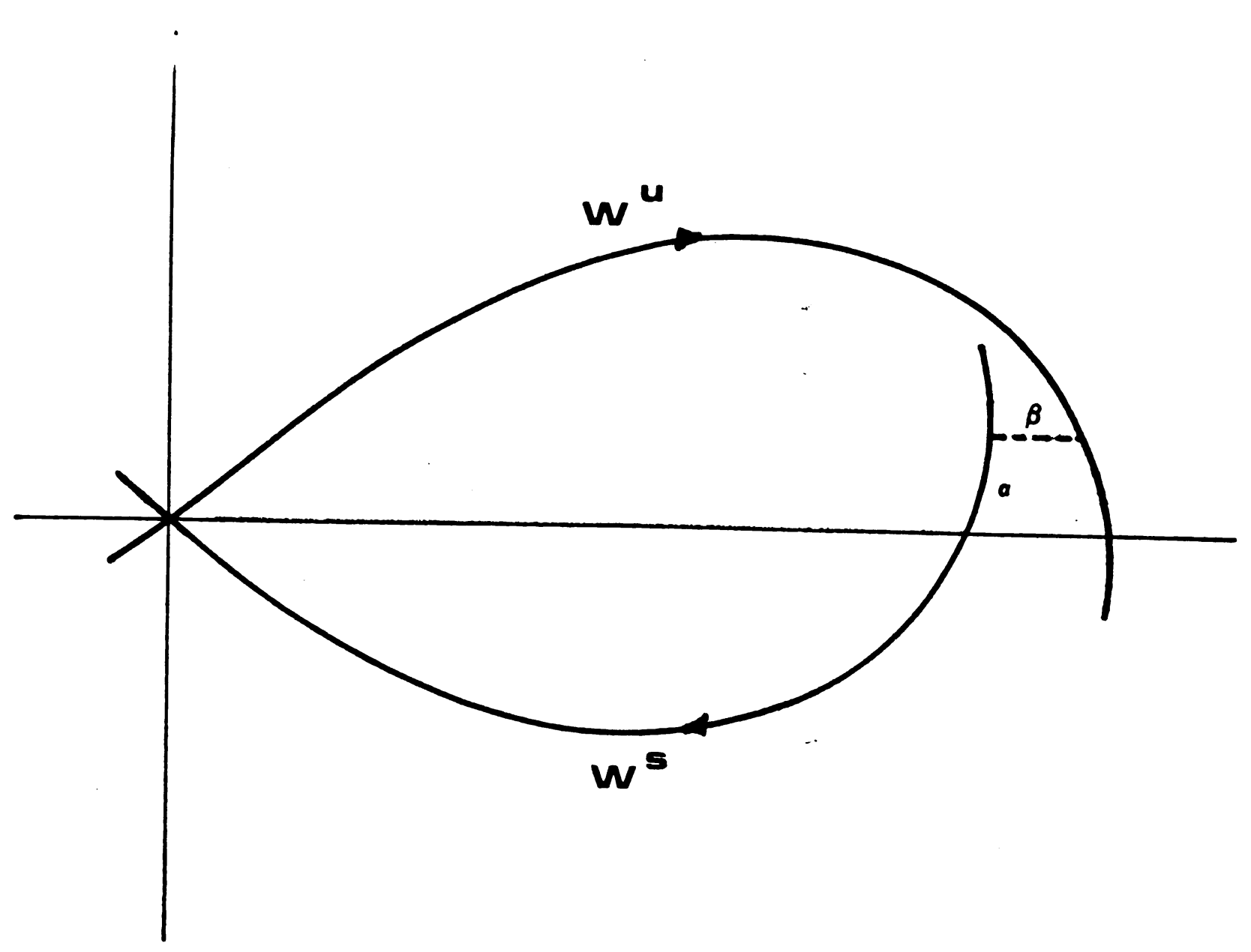

Figure 7: Stable manifold coordinates.

By the standard argument, ${ }^{4}$ one finds that

$$
d(f, \varepsilon)=\varepsilon \nu M(f)+0\left(\varepsilon^{2}\right)
$$

where $\nu=\|F(\bar{u})\|^{-1}$ is constant, and $M$ is the Melnikov functional

$$
M(f)=\int_{-\infty}^{\infty} F\left(u^{0}(t)\right) \wedge f\left(t, u^{0}(t)\right) d t
$$

Since $M(f)$ is linear in $f$, it is differentiable in $f \in \mathcal{G}$ By the standard argument one can show that the function $d(f, \varepsilon)$ is also differentiable in $f \in \mathcal{G}$ and $\varepsilon \in\left(-\varepsilon_{0}, \varepsilon_{0}\right)$ That is,

$$
d: H \times\left(-\varepsilon_{0}, \varepsilon_{0}\right) \rightarrow R
$$

is a $C^{1}$ function. Let $D_{1} d$ be the partial of $d$ with respect to its first argument so

$$
D_{1} d(f, \varepsilon)(g)=\varepsilon \nu M(g)+0\left(\varepsilon^{2}\right) .
$$

\footnotetext{
${ }^{4}$ Melnikov (1963) and Guckenheimer and Holmes (1983) are good references to the classical Melnikov theory used in this section.
} 
In the usual development in the periodic case the Melnikov functional depends on a real parameter. To see how our definition is the natural extension, let $H$ be the hull of a periodic function $p$, the restriction of $M$ to $H$ is

$$
M\left(p_{-\tau}\right)=\int_{-\infty}^{\infty} F\left(u^{0}(t)\right) \wedge p(t-\tau) d t,
$$

which is the usual Melnikov function in the periodic case. By allowing $f$ to vary over the full $H$, we see that our formulation includes the traditional version.

Since $F\left(u^{0}(t)\right)$ tends to zero exponentially fast as $t \rightarrow \pm \infty$, we see that $M: \mathcal{G} \rightarrow R$ as a bounded linear functional, which we call the Melnikov functional. Given $f \in \mathcal{G}$, we define the composition $\mathcal{M}(f)(t)=M\left(f_{t}\right)$, to be the Melnikov transform of $f$. Note that

$$
\begin{aligned}
\mathcal{M}(f)(t)=M\left(f_{t}\right) & =\int_{-\infty}^{\infty} F\left(u^{0}(s)\right) \wedge f\left(s+t, u^{0}(s)\right) d s \\
& =\int_{-\infty}^{\infty} F\left(u^{0}(\sigma-t)\right) \wedge f\left(\sigma, u^{0}(\sigma-t)\right) d \sigma
\end{aligned}
$$

Since $\mathcal{M}$ is a composition, it follows that if $f$ is almost periodic, then $\mathcal{M}(f)$ is also almost periodic.

In order to prove that the stable and unstable bundles intersect transversally we will require a sign change near some $(f, \varepsilon)$. This can be accomplished for small $\varepsilon$ by studying the zero set of the Melnikov functional $M$, which we define to be

$$
Z=\{f \in H: M(f)=0\} .
$$

We will say that the compact invariant set $H$ admits a simple Melnikov zero set $Z$, if $Z$ is nonempty and

$$
\left.\frac{d}{d t} M\left(f_{t}\right)\right|_{t=0} \neq 0
$$

for all $f \in Z$. By (3.9) this is equivalent to the Melnikov transform of $f$ having a simple zero at zero. Also by the second part of (3.9) it is clear that the derivative in (3.11) exists even if the original function $f$ is differentiable in $x$ but only continuous in $t$.

Later in this paper we will derive our main result which asserts that if $H$ admits a simple Melnikov zero set $Z$, then the skew product flow generated by (3.1) for $f \in H$, contains a Bernoulli bundle $\mathcal{B}_{n}(K)$ for every $n \geq 2$ and for every small $\varepsilon$. However we first want to derive an important consequence of the existence of a simple zero set.

Theorem 1. Let $H$ be a compact invariant set in $\mathcal{G}$. Assume that $H$ is a minimal set and that $H$ admits a simple (nonempty) zero set $Z$. Then $Z$ is a global cross section to the flow on $H$.

Proof. Since $H$ is compact and the Melnikov functional $M: H \rightarrow R$ is continuous, it follows that $Z$ is closed and therefore compact. By (3.11) if $f \in Z$ there is a $\delta>0$ such 
that $f_{t} \notin Z$ for $0<|t| \leq \delta$, and by the compactness of $Z, \delta$ can be chosen independent of $f \in Z$. Let $f \in Z$ and $g \in H$. By the above there is a $\delta>0$ such that $M\left(f_{-\delta}\right)$ and $M\left(f_{\delta}\right)$ are of opposite sign. Since the orbit of $g$ is dense in $H$ there is a time $\tau$ such that $g_{\tau}$ is arbitrary close to $f$. In particular we can find $\tau$ so that $M\left(g_{\tau-\delta}\right)$ and $M\left(g_{\tau+\delta}\right)$ are of opposite sign. Therefore for some $\tau_{0} \in(\tau-\delta, \tau+\delta)$, one has $g_{\tau_{0}} \in Z$. This proves that all orbits meet $Z .^{5}$ For $f \in Z$ define $T(f)>0$ to be the least time of return. The above argument also shows that $T$ is a continuous mapping, and thus $Z$ is a global cross section.

The fact that the zero set $Z$ is a global cross section to the flow on $H$ means that the Melnikov functional prescribes a synchronization—or internal clock-for the flow on $H$.

III.4 Proper Melnikov Perturbations. One of the main objectives in developing dynamical systems is to seek a theories which have some degree of robustness. In other words, one would like to develop a theory which will persist under an appropriate set of perturbations. Not all dynamical theories have this property, however many of the dynamical features we study in this work do. In order to do this it is necessary to present the concept of a proper Melnikov perturbation. Before doing this though let us look at an illustrative example.

Consider the equation

$$
\begin{aligned}
& x^{\prime}=F(x)+\varepsilon f(x, \theta) \\
& \theta^{\prime}=\omega
\end{aligned}
$$

where $x \in R^{2}, \theta \in T^{2}$ (the 2-dimensional forms), $F: R^{2} \rightarrow R^{2}$ and $f: R^{2} \times T^{2} \rightarrow R^{2}$ are smooth functions, $F$ satisfies the conditions stated in Section III.1 and $\omega=\left(\omega_{1}, \omega_{2}\right)$ is constant with $\omega_{1} / \omega_{2}$ irrational. This is an example of a quasi periodic perturbation of $x^{\prime}=F(x)$, and the theory we describe below will apply in this case.

Let us now introduce a small change in (3.12) by perturbing the $\theta$-equation to obtain:

$$
\begin{aligned}
x^{\prime} & =F(x)+\varepsilon f(x, \theta) \\
\theta^{\prime} & =\omega+\varepsilon g(x, \theta)
\end{aligned}
$$

where $g: R^{2} \times T^{2} \rightarrow T^{2}$ is smooth. The perturbation theory given in Sections III.1 and III.2 still apply in this case, see Hale (1969). In particular the invariant torus $H_{0}=\left\{v_{0}\right\} \times T^{2}$ for the unperturbed equation $(\varepsilon=0)$ becomes an invariant torus $H_{\varepsilon}$ for the perturbed equation when $\varepsilon$ is small and nonzeero. However the flow on $M_{\varepsilon}$ need not be quasi periodic. In fact it is generally not quasi periodic for $\varepsilon \neq 0$ because one expects a locking-in to occur and that the flow on the torus $H_{\varepsilon}$ will contain stable and unstable periodic orbits. Eventhough this example is not minimal, it is evident that the flow on $H_{\varepsilon}$ still has a global cross section.

\footnotetext{
${ }^{5}$ By using the Birkhoff recurrence property for minimal sets, we see that every orbit meets $Z$ infinitely often.
} 
Because of Theorem 1 and the considerations of the last paragraph we make the following definition: Consider equation (3.1) for $f \in H$, where $H$ is a compact invariant set in $\mathcal{G}$. Let $M: H \rightarrow R$ be the Melnikov functional given by (3.8). We shall say that $H$ is a proper Melnikov perturbation if there is a global cross section $Z$ to $H$ such that $Z \subset M^{-1}(0)$, i.e., $Z$ lies in the zero-set of the Melnikov functional, and for each $f \in Z$, inequality (3.11) is valid.

The following proposition shows that the property that compact invariant set $H \subset \mathcal{G}$ be a proper Melnikov perturbation is an open property in the class of compact invariant sets in $\mathcal{G}$. We shall use the symbol $B_{\delta}(H)$ to denote an $\delta$-neighborhood of $H$ in $\mathcal{G}$.

Proposition. Let $H$ be a compact invariant set in $\mathcal{G}$ which is a proper Melnikov perturbation. Then there is an $\delta>0$ with the property that every compact invariant set $\hat{H}$ satisfying

$$
\hat{H} \subset B_{\delta}(H), \quad H \subset B_{\delta}(\hat{H})
$$

is also a proper Melnikov perturbation.

The proof of this is straight forward application of the Implicit Function Theorem and we omit the details.

In the case of equation (3.13) one can show that for $\varepsilon$ sufficiently small that (3.14) is valid where $H=H_{0}$ and $\hat{H}=H_{\varepsilon}$. A special case of this is discussed in Section VI.

III.5 Duffing's Equation. Let us consider one of the standard examples in this subject, Duffing's equation with negative linear stiffness and almost periodic forcing:

$$
\begin{aligned}
& \dot{x}_{1}=x_{2} \\
& \dot{x}_{2}=x_{1}-x_{1}^{3}+\varepsilon f(t)
\end{aligned}
$$

where $x=\left(x_{1}, x_{2}\right), F(x)=\left(x_{2}, x_{1}-x_{1}^{3}\right)$ and $f: R \rightarrow R$ is almost periodic. Here we assume that $f$ is independent of $x$, i.e., $f$ does not add dissipation to Duffing's equation. When $\varepsilon=0$ this system has centers at $( \pm 1,0)$ and a non-degenerate saddle at the origin. The system is Hamiltonian with $H=\frac{1}{2}\left(x_{2}^{2}-x_{1}^{2}\right)+x_{1}^{4} / 4$ and so the phase portrait is easy to analyze, see Figure 1. There are two orbits homoclinic to the origin $v_{0}=(0,0)$. The homoclinic orbits which cross the $x_{1}$-axis at epoch $t=0$ are $\pm(\sqrt{2} \operatorname{sech} t,-\sqrt{2} \operatorname{sech} t \tanh t)$. Choose the right homoclinic orbit by taking the plus sign above. Since the Melnikov functional is linear in $f$ we need only compute it on sines and cosines. Residue calculus yields

$$
M(\cos (\omega t+a))=\sqrt{2} \pi \operatorname{sech}\left(\frac{\pi \omega}{2}\right) \sin a .
$$

Return to the example $(Q P)$ we let $H$ be the hull of the quasi periodic function

$$
q(t)=a_{1} \cos \omega_{1} t+a_{2} \cos \omega_{2} t
$$


where $a_{1}, a_{2}$ are nonzero and $\omega_{1} / \omega_{2}$ is irrational. Then by the results of the last section, the hull of $f$ is $H=\left\{a_{1} \cos \left(\omega_{1} t+\phi_{1}\right)+a_{2} \cos \left(\omega_{2} t+\phi_{2}\right)\right\}$, which is homeomorphic to $T^{2}=R^{2} /(2 \pi Z)^{2}$. By (3.16) one has

$$
M\left(a_{1} \cos \left(\omega_{1} t+\phi_{1}\right)+a_{2} \cos \left(\omega_{2} t+\phi_{2}\right)\right)=A_{1} \sin \phi_{1}+A_{2} \sin \phi_{2}
$$

where

$$
A_{i}=a_{i} \sqrt{2} \omega_{i} \operatorname{sech}\left(\frac{\pi \omega_{i}}{2}\right) .
$$

For fixed $\omega_{1}, \omega_{2} \neq 0$, it is clear that we can prescribe $A_{1}$ and $A_{2}$ and uniquely determine $a_{1}$ and $a_{2}$. Thus we can first choose the Melnikov function and hence its zero set and then find the function $f$, i.e., we can invert the Melnikov transform. Thus the zero set $Z$ is defined by $A_{1} \sin \phi_{1}+A_{2} \sin \phi_{2}=0$ and condition (3.11) becomes $\omega_{1} A_{1} \cos \phi_{1}+\omega_{2} A_{2} \cos \phi_{2} \neq 0$. The case when $A_{1}=-A_{2}$ gives $\phi_{1}=\phi_{2}$ and so $Z$ is the diagonal in Figure 2. In this case, condition (3.11) is satisfied since $\omega_{1} / \omega_{2}$ is irrational. ${ }^{6}$ One can use either angle as a coordinate system on the diagonal, so choose $\phi_{1}$. The orbit starting at $\left(\alpha_{1}, \alpha_{1}\right)$ is $\left(\alpha_{1}+\omega_{1} t, \alpha_{1}+\omega_{2} t\right)$; the return time is $T=2 \pi /\left|\omega_{1}-\omega_{2}\right|$; and the Poincare map is $\alpha_{1} \rightarrow \alpha_{1}+2 \pi\left|\omega_{1} / \omega_{1}-\omega_{2}\right|$, which is an irrational rotation of a circle.

Next turn to the limit periodic example (LP) and let $H$ be the hull of

$$
\ell(t)=\sum_{k=1}^{\infty} a_{k} \cos 2 \pi\left(t / 2^{k}\right)
$$

Thus $H$ consists of all functions of the form

$$
\ell^{*}(t)=\sum_{k=1}^{\infty} a_{k} \cos 2 \pi\left(\left(t+\phi_{k}\right) / 2^{k}\right)
$$

with $\phi_{k}=\phi_{k+1} \bmod 2^{k}$. Now by (3.16) one has

$$
M\left(\ell^{*}\right)=\sum_{k=1}^{\infty} A_{k} \sin 2 \pi\left(\phi_{k} / 2^{k}\right)
$$

where

$$
A_{k}=\sqrt{2} \pi a_{k} \operatorname{sech}\left(\pi^{2} 2^{k}\right) .
$$

Here we cannot prescribe the $A_{k}$ 's completely arbitrary as before, but is clear that we can choose non-zero $A_{k}$ 's such that the series (3.17), (3.18) and (3.19) all converge rapidly. By (3.16) one has

$$
g(\tau) \stackrel{\text { def }}{=} M\left(\ell_{\tau}^{*}\right)=\sum_{k=1}^{\infty} A_{k} \sin 2 \pi\left(\left(\tau+\phi_{k}\right) / 2^{k}\right),
$$

\footnotetext{
${ }^{6}$ More generally (3.11) would be valid provided the ratio $\omega_{1} / \omega_{2} \neq 1$.
} 
i.e., $g$ is a limit periodic function and the Melnikov transform of $\ell^{*}$. From (3.20) we see that $\ell^{*} \in Z$ if and only if $g(0)=0$. Furthermore the first return time for $\ell^{*}$ is the first positive zero of $g$. The condition (3.11) reduces to the assertion that the Melnikov transform $g$ have simple zeros for every $\ell^{*} \in H$.

By taking $A_{1}=1$ and the remaining $A$ 's small, it is clear that one can construct a limit periodic function $g$ of the form (3.20) such that it and everything in its hull has simple zeros. Moreover, the $A$ 's can be choosen so small that the corresponding series (3.17) converges uniformly so that $\ell$ is a limit periodic function.

III.6 Transversal Intersections. If $Z \subset H$ is a cross section for the translational flow $\pi$ on $H$ with first return time $T: Z \rightarrow R$, then $S=R^{2} \times Z$ is a cross section for the skew product flow $\Pi$ on $R^{2} \times H$ with the same first return time, and with Poincaré map given by

$$
\Psi=(\psi, \eta): S \rightarrow S:\left(x^{0}, f\right) \rightarrow\left(\psi\left(x_{0}, f, \varepsilon\right), \eta(f)\right),
$$

where

$$
\psi\left(x_{0}, f, \varepsilon\right)=\phi\left(T(f), x_{0}, f, \varepsilon\right), \quad \eta(f)=f_{T(f)} .
$$

Note that $\Psi$ is a discrete skew product dynamical system on $R^{2} \times Z$ for $|\varepsilon|<\varepsilon_{0}$.

When $\varepsilon=0$ the solutions of (3.1) do not depend on $f$ and the skew product becomes an ordinary product. For example if $f$ is quasi periodic in $t$, then the flow, $\Pi$ is the product of the irrational twist flow on the torus, as pictured in Figure 2, and the Duffing flow as pictured in Figure 1. In the limit periodic case the flow $\Pi$ is the product of the solenoidal flow and the Duffing flow.

It may by easier to visualize the Poincare map (3.21). First consider the Example (QP) with $f=q$ when $\varepsilon=0$. The cross section of the translational flow is a circle, for example the diagonal of Figure 2, and the Poincare map on $H$ is an irrational rotation of the circle with return time $T$. Now integrate Duffing's equation for a time $T$ to obtain a map of the plane as shown in Figure 1. Figure 8 tries to illustrate the product map (3.21) at $\varepsilon=0$. The $\alpha$ axis coming out of the plane of the paper is an angular variable and should be identified $\bmod 2 \pi$ since the space is $R^{2} \times S^{1}$. The map carries a plane perpendicular to the $\alpha$-axis into another such plane. (This is true for small $\varepsilon \neq 0$ also since the second term in (3.21) is always the translational flow). The $\alpha$-axis is an invariant circle for the Poincare map $\Psi$ and its stable and unstable manifolds are the products of the figure eight and a circle. One of the two loops of the figure eight is illustrated in Figure 8.

For the limit periodic example (LP) with $f=\ell$ and $\varepsilon=0$, one must use some imagination. Think of a Cantor set along the $\alpha$-axis in Figure 8. The Poincare map $\Psi$ is similar to the above except the Cantor set of planes perpendicular to the $\alpha$-axis are shuffled by the adding machine map.

The main result of this section is the following: 


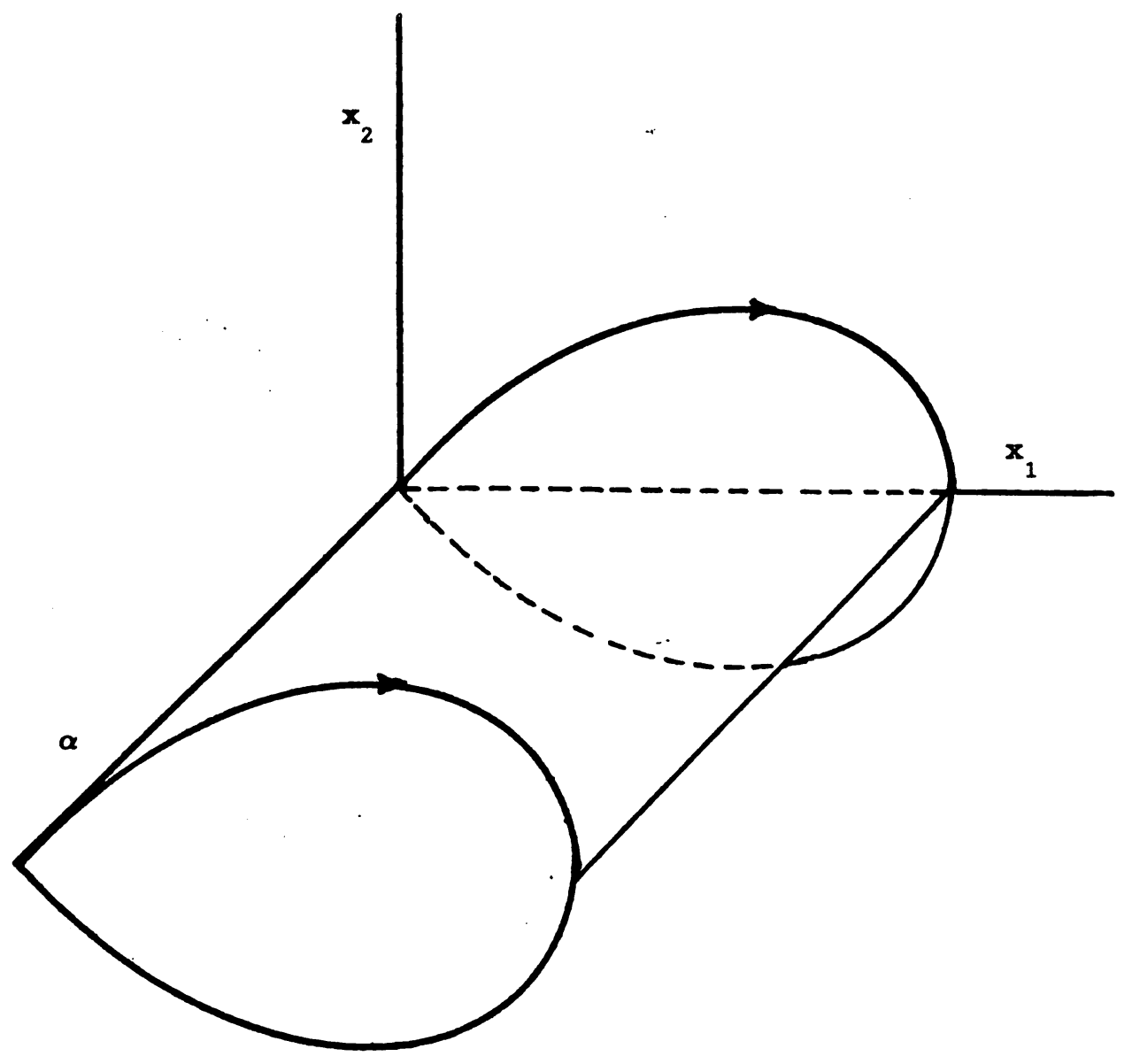

Figure 8: The unperturbed dynamics

THEOREM 2. Let (3.1) be given where $f \in H$ and $H$ is a minimal set in $\mathcal{G}$ that admits a simple zero set $Z=M^{-1}(0)$. Then there is an $\varepsilon_{0}$ such that for every $\varepsilon$ with $|\varepsilon|<\varepsilon_{0}$ and for each $f \in Z$ there is a unique point

$$
\xi(f, \varepsilon)=\bar{u}+O(\varepsilon) \in R^{2}
$$

such that $(\xi(f, \varepsilon), f) \in \mathcal{W}^{s}(\varepsilon) \cap \mathcal{W}^{u}(\varepsilon)$ for $0<|\varepsilon|<\varepsilon_{0}$. The function $\xi$ is continuous. Moreover, if $\Xi^{0}=\{(\xi(f, \varepsilon), f): f \in Z\}$ and $\Xi^{k}=\Psi^{k}\left(\Xi^{0}\right)$, then

$$
\Lambda=V \cup\left\{\bigcup_{-\infty}^{\infty} \Xi^{k}\right\}
$$

is a compact invariant set for the Poincaré map $\Psi$. For $\varepsilon \neq 0$, each point of $\Xi^{k}$ is a point of transversal intersection of the stable and unstable manifolds of $V$.

The same conclusions hold if $H$ is a compact invariant set in $\mathcal{G}$ that is a proper Melnikov perturbation.

Proof. As described in Section III.3, the separation of the stable and unstable manifold 
in the normal direction $n$ is

$$
\begin{aligned}
d(f, \varepsilon) & =\left\|u_{s}(0, f, \varepsilon)-u_{u}(0, f, \varepsilon)\right\| \\
& =\varepsilon \nu M(f)+O\left(\varepsilon^{2}\right)
\end{aligned}
$$

where $\nu$ is a nonzero constant. We define a coordinate system near $\bar{u}$ in $R^{2}$ as follows: Consider the map $(\alpha, \beta) \rightarrow u_{s}(\alpha, f, \varepsilon)+\beta n$, where $n$ is now a unit normal vector to $F(\bar{u})$, see Figure 7. This map takes a neighborhood of the origin in $R^{2}$ onto a neighborhood of $\bar{u}$. Note that $\alpha$ is a coordinate along the stable manifold (essentially the time parameter) and $\beta$ is a coordinate in the unit normal direction. By taking the Jacobian of this map when $\alpha=\beta=\varepsilon=0$ and using the fact that $n$ is normal to $F(\bar{u})$, one sees that the Jacobian is nonsingular and $(\alpha, \beta)$ constitutes a valid coordinate system near $\bar{u}$ when $\varepsilon$ is small.

In this coordinate system the stable manifold has coordinates $(\alpha, 0)$. The unstable manifold

$$
\begin{aligned}
u_{u}(\alpha, f, \varepsilon) & =u_{s}(\alpha, f, \varepsilon) \pm d\left(f_{\alpha}, \varepsilon\right) n \\
& =u_{s}(\alpha, f, \varepsilon) \pm \varepsilon \nu M\left(f_{\alpha}\right)+0\left(\varepsilon^{2}\right)
\end{aligned}
$$

has coordinates $\left(\alpha, \pm \varepsilon \nu M\left(f_{\alpha}\right)+0\left(\varepsilon^{2}\right)\right){ }^{7}$ In these coordinates an intersection of the stable and unstable manifold is obtained when $\varepsilon \nu M\left(f_{\alpha}\right)+0\left(\varepsilon^{2}\right)=0$, that is when $G(\alpha, f, \varepsilon)=0$ where $G(\alpha, f, \varepsilon)=M\left(f_{\alpha}\right)+0(\varepsilon)$. By assumption one has

$$
G(0, f, 0)=M(f)=0, \quad D_{1} G(0, f, 0) \neq 0
$$

when $f \in Z$. Thus by the Implicit Function Theorem there is an $\varepsilon_{0}>0$ such that one can find a solution $\alpha=\bar{\alpha}(f, \varepsilon)$ of $G(\alpha, f, \varepsilon)=0$ for $f \in Z$ and $|\varepsilon|<\varepsilon_{0}$. In these coordinates the intersection of the stable and unstable manifold is precisely $(\alpha, \bar{\alpha}(f, \varepsilon))$, which we define to be $\xi(f, \varepsilon)$. The intersection is clearly transversal since the zeros of $M\left(f_{t}\right)$ are simple for $f \in Z$

Since $(\xi(f, \varepsilon), f)$ lies in both the stable and unstable bundles for $V$, one has

$$
P^{k}(\xi,(f, \varepsilon), f) \rightarrow V \text { as } k \rightarrow \pm \infty
$$

Since $(\xi(f, \varepsilon), f)$ is a typical point in $\Xi^{0}$ this shows that $\Lambda=V \cup\left\{\bigcup_{k=-\infty}^{\infty} \Xi^{k}\right\}$ is closed and hence compact. Clearly $\Lambda$ is invariant. Since $\xi(f, \epsilon), f,) \in \Xi^{0}$ is a point of transversal intersection of the stable and unstable manifolds for $V$, and since the Poincare mapping $P$

\footnotetext{
${ }^{7}$ The sign in (3.22) is + if the inner product

$$
\left\langle n,\left[u_{u}(0, f, \varepsilon)-u_{s}(0, f, \varepsilon)\right]\right\rangle
$$
}

is positive, otherwise the sign is minus. 
preserves this property we sese that each point of $\Xi^{k}$ is a point of transversal intersection of the stable and unstable manifolds of $V$.

The argument for a compact invariant set $H$, which is a proper Melnikov perturbation, is identical.

Remark.. A related construction of homoclinic orbits can be found in Chow, Hale and Mallet-Paret (1980).

\section{Hyperbolic Sets and the Shadowing Lemma}

In this section we shall concentrate on the Poincaré map considered as a discrete skew product dynamical system and develop the skew product analogs of hyperpolic sets and the Shadowing Lemma, see Bowen (1970) and Conley (1978). Also in this section the notation of the last section will be used in a slightly more general setting since we will take the second factor to be an arbitrary discrete dynamical system. Let

$$
\Psi=(\psi, \eta): R^{n} \times Z \rightarrow R^{n} \times Z:(x, f) \rightarrow(\psi(x, f), \eta(f))
$$

define a discrete skew product dynamical system, where $Z$ is an arbitrary compact Hausdorff space, This means that both $\Psi$ and $\eta$ are homeomorphisms. Furthermore, assume that $\Psi$ and $\psi$ are smooth in their first argument, i.e., $D_{1} \psi^{k}(x, f)$ is defined and continuous for all $(x, f) \in R^{n} \times Z$. For each integer $k$ we define $\Psi(x, f, k): R^{n} \times Z \rightarrow R^{n}$ by

$$
\Psi^{k}(x, f)=\left(\Psi(x, f, k), \eta^{k}(f)\right)
$$

Since one has

$$
\Psi(x, f, k+1)=\Psi\left(\Psi(x, f, k), \eta^{k}(f), 1\right)
$$

for all $k$, a simple induction argument shows that the derivative $D_{1} \Psi(x, f, k)$ exists and is continuous for all $(x, f) \in R^{n} \times Z$.

IV.1 Skew Hyperbolic Sets and Exponential Dichotomies. There is a linear skew product flow associated with (4.1). Let $p=(x, f)$ denote a typical point in $R^{n} \times Z$. Define $\Phi(p, k)$ to be the linear mapping

$$
\Phi(p, k) u=\Psi(x, f, k) u
$$

where $p=(x, f)$ and $k$ is an integer. Then the mapping

$$
\Pi(u, p, k)=\left(\Phi(p, k) u, \Psi^{k}(p)\right)
$$

defines a linear skew product flow over $R^{n} \times Z$. Let $\Lambda \subset R^{n} \times Z$ be a compact invariant set for the mapping $\Psi$. Following Sacker and Sell (1974) one says that $\Phi$ admits an 
exponential dichotomy over $\Lambda$ if there is a projector $(u, p) \rightarrow(P(p) u, p)$ and positive constants $K, \alpha$ such that

$$
\begin{cases}\|\Phi(p, k) P(p)\| \leq K e^{-\alpha k}, & p \in \Lambda, k \geq 0 \\ \|\Phi(p, k)[I-P(p)]\| \leq K e^{\alpha k}, & p \in \Lambda, k \leq 0\end{cases}
$$

Recall that a projector $(u, p) \rightarrow(P(p) u, p)$ is a continuous mapping of $R^{n} \times \Lambda$ into itself with the property that $P(p)$ is a linear projection on $R^{n}$ for every $p \in \Lambda$.

We shall also say that $\Lambda$ is skew hyperbolic if $\Phi$ admits an exponential dichotomy over $\Lambda$. The exponential dichotomy also asserts that there is a continuous splitting $R^{n}=$ $E_{p}^{s}+E_{p}^{u}, p \in \Lambda$, where

$$
E_{p}^{s}=\text { Range } P(p) \quad \text { and } \quad E_{p}^{u}=\text { Null Space } P(p) \text {. }
$$

It follows from (4.3) that if $u \in E_{p}^{s}$, then

$$
\|\Phi(p, k) u\| \leq K e^{-\alpha k}\|u\|, \quad k \geq 0
$$

While if $u \in E_{p}^{u}$ then

$$
\|\Phi(p, k) u\| \leq K e^{\alpha k}\|u\|, \quad k \leq 0
$$

Also note that the projection $P(p)$ satisfies the identity

$$
P\left(\Psi^{k}(p)\right)=\Phi(p, k) P(p) \Phi^{-1}(p, k), \quad \quad p \in \Lambda
$$

for every integer $k$.

Let us now return to the invarient set $\Lambda=V \cup\left\{\bigcup_{-\infty}^{\infty} \Xi^{k}\right\}$ described in the last section.

Theorem 3. Assume that the hypotheses of Theorem 2 are satisfied, i.e., $H$ is a minimal set in $\mathcal{G}$ that admits a simple zero set $Z=M^{-1}(0)$. Let $\Lambda$ be given by Theorem 2. Let $\Psi=(\psi, \eta)$ be the Poincaré map induced on the section $S=R^{2} \times Z$ and let $\Phi$ be the linearized flow given by (4.2). Then $\Phi$ admits an exponential dichotomy over $\Lambda$.

The same conclusion is valid if $H$ is a compact invariant set in $\mathcal{G}$ that is a proper Melnikov perturbation.

Proof. This proof is somewhat standard so we will refer the reader to the literature at several points. Using the notation of section III, let $\phi\left(t, x_{0}, f, \varepsilon\right)$ be the solution of (3.1) which satisfies $\phi\left(0, x_{0}, f, \varepsilon\right)=x_{0}$; let $Z \subset H$ be the cross section of the translation flow defined by $Z=M^{-1}(0)$; let $T: Z \rightarrow R$ the first return time; let $\psi\left(x_{0}, f, \varepsilon\right)=$ $\phi\left(T(f), x_{0}, f, \varepsilon\right)$; and let

$$
\Psi=(\psi, \eta): S \times I \rightarrow S:\left(x_{0}, f, \varepsilon\right) \rightarrow\left(\psi\left(x_{0}, f, \varepsilon\right), \eta(f)\right)
$$


where $I=\left(-\varepsilon_{0}, \varepsilon_{0}\right)$ and $S=R^{2} \times Z$.

By Theorem 2 of the previous section, at each point $p \in \Xi^{k}$ the stable and unstable manifolds intersect transversally; that is, if we define $E_{p}^{s}=T_{p} \mathcal{W}^{s}(\varepsilon) \cap S$ and $E_{p}^{u}=T_{p} \mathcal{W}^{u}(\varepsilon) \cap S$ as in formula (3.7), then by the definition of transversality $T_{p} R^{2}=$ $R^{2}=E_{p}^{s}+E_{p}^{u}$ and the splitting is continious by the smoothness of the stable and unstable manifolds. If $p \in V \cap S$, then by the same definition and the local stable manifold theorem as given for example in Hale (1969) one has $T_{p} R^{2}=E_{p}^{s}+E_{p}^{u}$.

The estimates in (4.3) follow at once from the general properties of exponential dichotomies as given in Coppel (1978) and as explicitly shown in the proof of Proposition 2.2 of Palmer (1984) for the periodic case. We note that the results given in Coppel (1978) do not require the system to be periodic and so Palmer's argument hold in the present case also.

IV.2 The Skew Shadowing Lemma. The first concept we need for the Shadowing Lemma required is that of skew pseudo orbit. As in the case of a pseudo orbit, a skew pseudo orbit this is a bisequence in $\Lambda$ that is close to an actually orbit however the jumps are permitted only in the $x$-variable not in $f$. More precisely for $\alpha>0$ a (skew) $\alpha$-pseudo orbit for $\Psi \mid \Lambda$ is a bisequence $\left\{p_{i}=\left(x_{i}, f_{i}\right)\right\}, i$ ranging from $-\infty$ to $+\infty, p_{i} \in \Lambda$, such that $f_{i+1}=\eta\left(f_{i}\right)$ and $d\left(\psi\left(x_{i}, f_{i}\right), x_{i+1}\right)<\alpha$ for all $i$. Thus the sequence $\left\{f_{i}\right\}$ is an $\eta$-orbit but the components in $R^{n}$ may jump by as much as $\alpha$.

One says that a $\Psi$ orbit $\left\{\Psi^{i}(y, f)=\left(y_{i}, f_{i}\right)\right\}$ is a $\beta$-shadow for an $\alpha$-pseudo orbit $\left\{\left(x_{i}, f_{i}\right)\right\}$ if $d\left(x_{i}, y_{i}\right)<\beta$ and $f_{i+1}=\eta\left(f_{i}\right)$ for $i$. Note that the base orbits are the same. In this context we have:

ThEOREM 4. (The Skew Shadowing Lemma): Let $\Lambda$ be a compact, skew hyperbolic invariant set for $\Psi: R^{n} \times Z \rightarrow R^{n} \times Z$. For every $\beta>0$ there is an $\alpha>0$ such that every $\alpha$-pseudo orbit for $\Psi \mid \Lambda$ is $\beta$-shadowed by some $\Psi$ orbit $\left\{\Psi^{i}(q)\right\}$. Moreover, there is a $\beta_{0}>0$ such that if $0<\beta<\beta_{0}$ then the $\Psi$-orbit given above is uniquely determined by the $\alpha$-psuedo orbit.

Proof. This is a slight variation of the proof found in Meyer and Sell (1987a). By modifying the functions $F$ and $f$ outside a compact neighborhood of the invariant set $\Lambda$ we may assume thet all orbits of $\Psi$ are bounded.

The following lemma will be useful for our proof: It asserts that the constant $K$ appearing in (4.3) can be chosen to be 1 .

Lemma. Assume that $\Phi$ admits an exponential dichotomy over $\Lambda$ and that (4.3) holds. Then there is a norm $\|\cdot\|_{p}$ on $R^{n}, p \in \Lambda$, with the property that

$$
\begin{cases}\|\Phi(p, k) P(p)\|_{p \cdot k} \leq e^{-\alpha k}, & k \geq 0 \\ \|\Phi(p, k)[I-P(p)]\|_{p \cdot k} \leq e^{\alpha k}, & k \leq 0\end{cases}
$$


where $p \cdot k=\Psi^{k}(p)$.

Proof. For any $(x, p) \in R^{n} \times \Lambda$ we have a unique decomposition $x=u+v$ where $u \in \operatorname{Range}(P(p))$ and $v \in$ Null space $(P(p))$. Now define $\|x\|_{p}=\max \left\{\|u\|_{p},\|v\|_{p}\right\}$ where

$$
\begin{aligned}
& \|u\|_{p}=\sup \left\{K^{-1} e^{\alpha k}\|\Phi(p, k) u\|: k \geq 0\right\} \\
& \|v\|_{p}=\sup \left\{K^{-1} e^{\alpha k}\|\Phi(p, k) v\|: k \leq 0\right\}
\end{aligned}
$$

The verification that (4.4) holds is straight forward and we omit the details.

In order to simplify our notation we shall drop the subscript $p \cdot k$ on the norms in (4.4). The context will make it clear which subscript is appropriate.

Let $\mathcal{M}=\ell_{\infty}$ be the Banach space of bounded bisequences

$$
x=\left\{x_{i}\right\}=\left\{\ldots, x_{-1}, x_{0}, x_{1}, \ldots\right\}, \quad x_{i} \in R^{n}
$$

with the supremum norm $\|x\|=\sup _{i}\left\|x_{i}\right\|$ and let $Z$ denote the space of $\eta$-orbits in $Z$, i.e.,

$$
Z=\left\{f=\left(\ldots, f_{-1}, f_{0}, f_{1}, \ldots\right) \in \times_{-\infty}^{\infty} Z: f_{i+1}=\eta\left(f_{i}\right)\right\}
$$

with the usual product topology. Define a map $\mathcal{F}: \mathcal{M} \times \mathcal{Z} \rightarrow \mathcal{M}$ by

$$
(\mathcal{F}(x, f))_{i+1}=\left(\psi\left(x_{i}, f_{i}\right)\right)
$$

and consider $f$ as a parameter. With $f$ fixed, $x$ is a fixed point of this map if and only if

$$
x_{i+1}=\psi\left(x_{i}, f_{i}\right), \quad i=0, \pm 1, \pm 2, \ldots
$$

or $\left\{\left(x_{i}, f_{i}\right)\right\}$ is a $\Psi$-orbit. Also $(x, f) \in \mathcal{M} \times Z$ is an $\alpha$-pseudo orbit if and only if $\| \mathcal{F}(x, f)-$ $x \|<\alpha$. The $\Psi$-orbit $\left\{\left(y_{i}, f_{i}\right)\right\} \beta$-shadows an $\alpha$-pseudo orbit $\left\{\left(x_{i}, f_{i}\right)\right\}$ if and only if $\mathcal{F}(y, f)=y$ and $\|x-y\|<\beta$, (Recall that the $\beta$-shadow and the $\alpha$-psuedo orbit must have the same $\eta$-orbit $\left.\left\{f_{i}\right\}\right)$. Let

$$
\mathcal{L}=\left\{(x, f) \in \mathcal{M} \times Z: x_{i} \in \Lambda \text { for all } i\right\}
$$

Thus, in this context the Shadowing Lemma says: For every $\beta>0$ there is a $\alpha>0$ such that if $(x, f) \in \mathcal{L}$ and $\|\mathcal{F}(x, f)-x\|<\alpha$, then there is a $(y, f) \in \mathcal{M} \times \mathcal{Z}$ such that $\mathcal{F}(y, f)=y$ and $\|x-y\|<\beta$. In other words, if $\|\mathcal{F}(x, f)-x\|<\alpha$ then $\mathcal{F}$ has a fixed point $y$ in an $\alpha$-neighborhood of $x$. This formulation suggest the use of the Inverse Function Theorem with the standard estimates on the domain of the inverse function.

The function $\mathcal{F}$ is differentiable with respect to its first argument with derivative given by

$$
\left(D_{1} \mathcal{F}(x, f)(w)\right)_{i}=D_{1} \psi\left(x_{i-1}, f_{i-1}\right)\left(w_{i}\right)
$$


where $x, w \in \mathcal{M}$. Let $x \in \mathcal{L}$ and define

$$
S_{x}=\times_{-\infty}^{\infty} E_{x_{i}}^{s} \quad U_{x}=\times_{-\infty}^{\infty} E_{x_{i}}^{u} .
$$

Since the original splitting is continuous, both $S_{x}$ and $U_{x}$ are closed, linear complimentary subspaces of $\mathcal{M}$ for each $x \in \mathcal{L}$. Consequently one has $T_{x} \mathcal{M}=S_{x}+U_{x}$ where $T_{x}$ denotes the tangent space at $x$. Also, $D \mathcal{F}(x)$ maps $S_{x}$ into $S_{\mathcal{F}(x)}$ and $U_{x}$ into $U_{\mathcal{F}(x)}$.

Using these facts we shall show that there is a constant $K_{0}$ such that

$$
\left\|D_{1} \mathcal{F}(x, f)-I\right\| \leq K_{0} \text { and }\left\|\left(D_{1} \mathcal{F}(x, f)-I\right)^{-1}\right\| \leq K_{0}
$$

for all $(x, f) \in \mathcal{L}$. The first estimate follows the fact that $D_{1} \psi$ is uniformly bounded on the compact set $\Lambda$. Because of linearity, the second estimate needs only be proved on the two complimentary subspaces $S_{x}$ and $U_{x}$. For $u \in S_{x}$ we have $\mu=e^{-\alpha}$ (see (4.4))

$$
\begin{aligned}
\left\|D_{1} \mathcal{F}(x, f)(u)-u\right\| & \geq\|u\|-\left\|D_{1} \mathcal{F}(x, f)(u)\right\| \\
& \geq\|u\|-\mu\|u\|=(1-\mu)\|u\|
\end{aligned}
$$

Hence $\left\|\left.\left(D_{1} \mathcal{F}(x, f)-I\right)\right|_{U_{s}} ^{-1}\right\| \leq(1-\mu)^{-1}$.

Since the linear skew product flow $\Pi$ is invertible on $R^{n} \times \Lambda$ there is a constant $K_{3}>0$ such that $\|\Phi(p, 1) w\| \geq K_{3}\|w\|$ for all $p \in \Lambda$ and $w \in R^{n}$. In particular this implies that $\left\|D_{1} \mathcal{F}(x, f)(v)\right\| \geq K_{3}\|v\|$ for all $(x, f) \in \mathcal{L}$ and $v \in U_{x}$. Let $v \in U_{x}$, then from (4.4) one has

$$
\begin{aligned}
\left\|D_{1} \mathcal{F}(x, f)(v)-v\right\| & =\left\|D_{1} \mathcal{F}(x, f)\left\{v-D_{1} \mathcal{F}^{-1}(x, f)(v)\right\}\right\| \\
& \geq K_{3}(\|v\|-\mu\|v\|)=K_{3}(1-\mu)\|v\|
\end{aligned}
$$

Hence $\left\|\left.\left(D_{1} \mathcal{F}(x, f)-I\right)\right|_{U_{z}} ^{-1}\right\| \leq K_{3}^{-1}(1-\mu)^{-1}$, which implies the second estimate in (4.5).

The following version of the inverse function theorem with estimate can be found in Hartman (1964). Let $B$ be a Banach space, $B_{\delta}(x)$ the ball of radius $\delta$ about $x \in B$, $G: B_{\delta}(x) \rightarrow B$ a $C^{1}$ function and $y_{0}=G\left(x_{0}\right)$.

InVERSE FunCtion TheOREM. Assume $D G(x)$ has a bounded inverse for all $x \in$ $B_{\delta}\left(x_{0}\right)$ with

$$
\|D G(x)\| \leq K \text { and }\left\|D G^{-1}(x)\right\| \leq K
$$

for all $x \in B_{\delta}\left(x_{0}\right)$ where $K$ is a constant. Let $p=\delta / K^{-2}$ and $q=\delta / K$, then there exists a domain $\Omega$, with $B_{p}\left(x_{0}\right) \subset \Omega \subset B_{\delta}\left(x_{0}\right)$ and such that $G$ is one-to-one on $\Omega$. Moreover, $B_{q}\left(y_{0}\right) \subset G(D)$.

Apply this version of the inverse function theorem to the function $\mathcal{G}(x, f)=\mathcal{F}(x, f)-x$ and consider $f \in Z$ as a parameter. Since $\psi$ is at least $C^{2}$ in its first argument and $\Lambda$ is 
compact the estimates in (4.5) can be extended to a neighborhood $\mathcal{N}$ of $\mathcal{L}$. In particular there is a $\delta_{0}>0$ such that $B_{\delta_{0}}(x) \subset \mathcal{N}$ for all $x \in \mathcal{L}$ and (4.5) holds on $B_{\delta_{0}}(x)$. Let $\alpha<\delta_{0}$ so the estimates (4.5) on the derivative of $\mathcal{F}$ hold on $B_{\alpha}(x)$ for $(x, f) \in \mathcal{N}$. Define $\beta=\alpha / K$. Then the inverse function theorem gives $\mathcal{G}\left(B_{\alpha}(x), f\right) \subset B_{\beta}(\mathcal{G}(x, f))$. Hence, if $\|\mathcal{G}(x, f)\|=\|\mathcal{F}(x, f)-x\|<\beta$, then $0 \in B_{\beta}(\mathcal{G}(x, f))$ or $0 \in \mathcal{G}\left(B_{\alpha}(x), f\right)$. Thus there is a $y \in B_{\alpha}(x)$ such that $\mathcal{G}(y, f)=\mathcal{F}(y, f)-y=0$. That is $(y, f)$ is an $\Psi$-orbit that $\alpha$-shadows the $\beta$-pseudo orbit $(x, f)$.

If we take $\beta_{0}=\delta_{0} / K^{2}$ then the inverse function theorem yields that $\mathcal{G}$ is one-to-one on $B_{\beta_{0}}(x)$ for $x \in \mathcal{L}$. Thus the distance between zeros of $\mathcal{G}$ is at least $\beta_{0}$ and this proves the uniqueness part of the Shadowing Lemma.

Remark. By the uniformity of the estimates given above the $\Psi$-orbits constructed here have a form of distality. That is, if $(x, f)$ and $(\bar{x}, f)$ are $\Psi$-orbits constructed above with $x \neq \bar{x}$ then $\|x-\bar{x}\|>\beta_{0}$ so for some $i$,

$$
\left\|x_{i}-\bar{x}_{i}\right\|=\left\|\psi^{i}\left(x_{0}, f_{0}\right)-\psi^{i}\left(\bar{x}_{0}, f_{0}\right)\right\|>\beta_{0} .
$$

\section{The Shadowing Lemma Establishes Bernoulli Bundles}

Henceforth, assume that $H$ is a compact invariant set in $\mathcal{G}$ and that $H$ is a proper Melnikov perturbation with global cross section $Z \subset M^{-1}(0) \subset H$. Thus for small $\varepsilon, \Lambda=$ $V \cup\left\{\bigcup_{-\infty}^{\infty} \Xi^{k}\right\}$ as defined in Theorems 2 and 3 is a hyperbolic invariant set for the Poincare map $\Psi=(\psi, \eta)$. Let $K$ be the transition matrix given by (2.7) and $A: B_{n}(K) \rightarrow B_{n}(K)$ the subshift of finite type which is defined by $K$. (The size $n$ of $K$ will be given within the proof of Theorem 5 below.) Let $A \times \eta: B_{n}(K) \times Z \rightarrow B_{n}(K) \times Z$ be the product map defined by (2.8) and let $A \otimes \eta$ denote the induced flow on the Bernoulli bundle $B_{n}(K) \otimes Z$.

TheOrem 5. Let the above assumptions be satisfied. There is an $\varepsilon_{0}>0$ such that for every $\varepsilon$ with $0<|\varepsilon|<\varepsilon_{0}$ there is an integer $n_{0}$ such that for all $n \geq n_{0}$, there is a compact invariant set $\Omega_{n} \subset R^{2} \times Z$ for the Poincaré map $\Psi$ such that the restriction $\Psi \mid \Omega_{n}$ is equivalent to the product map $A \times \eta$.

Proof. Assume that $\varepsilon_{0}>0$ is so small that the conclusions of Theorems 2 and 3 hold for $0<|\varepsilon|<\varepsilon_{0}$. Hence $\Lambda=V \cup\left\{\bigcup_{-\infty}^{\infty} \Xi^{k}\right\}$ is a compact hyperbolic set for the Poincaré map $\Psi: R^{2} \times Z \rightarrow R^{2} \times Z$. Let $\beta \leq \beta_{0}$ be as given in the uniqueness part of the shadowing lemma. We assume $\beta$ is small enough that $\operatorname{dist}\left(\Xi^{0}, \Xi^{i}\right)>4 \beta$ for $i \neq 0$; and let $\alpha$ be determined by the Skew Shadowing Lemma. Further restrict $\varepsilon_{0}$ so that by Theorem 0 for all $0<|\varepsilon|<\varepsilon_{0}$ the invariant set $V$ is within $\alpha / 4$ of $\{0\} \times Z$. Since $\Xi^{k} \rightarrow V$ as $k \rightarrow \pm \infty$ there is a $\kappa>0$ such that $\mathrm{d}$ ist $\left(\Xi^{k}, V\right)<\alpha / 4$ when $|k|>\kappa$. Let $N$ be the $\frac{\alpha}{2}$-neighborhood of $V$.

In order to simplify the proof somewhat we will relabel some of the $\Xi^{k}$ with subscripts as follows: As stated above $\Xi^{-\kappa}$ lies inside the neighborhood $N$. Since it will represent 


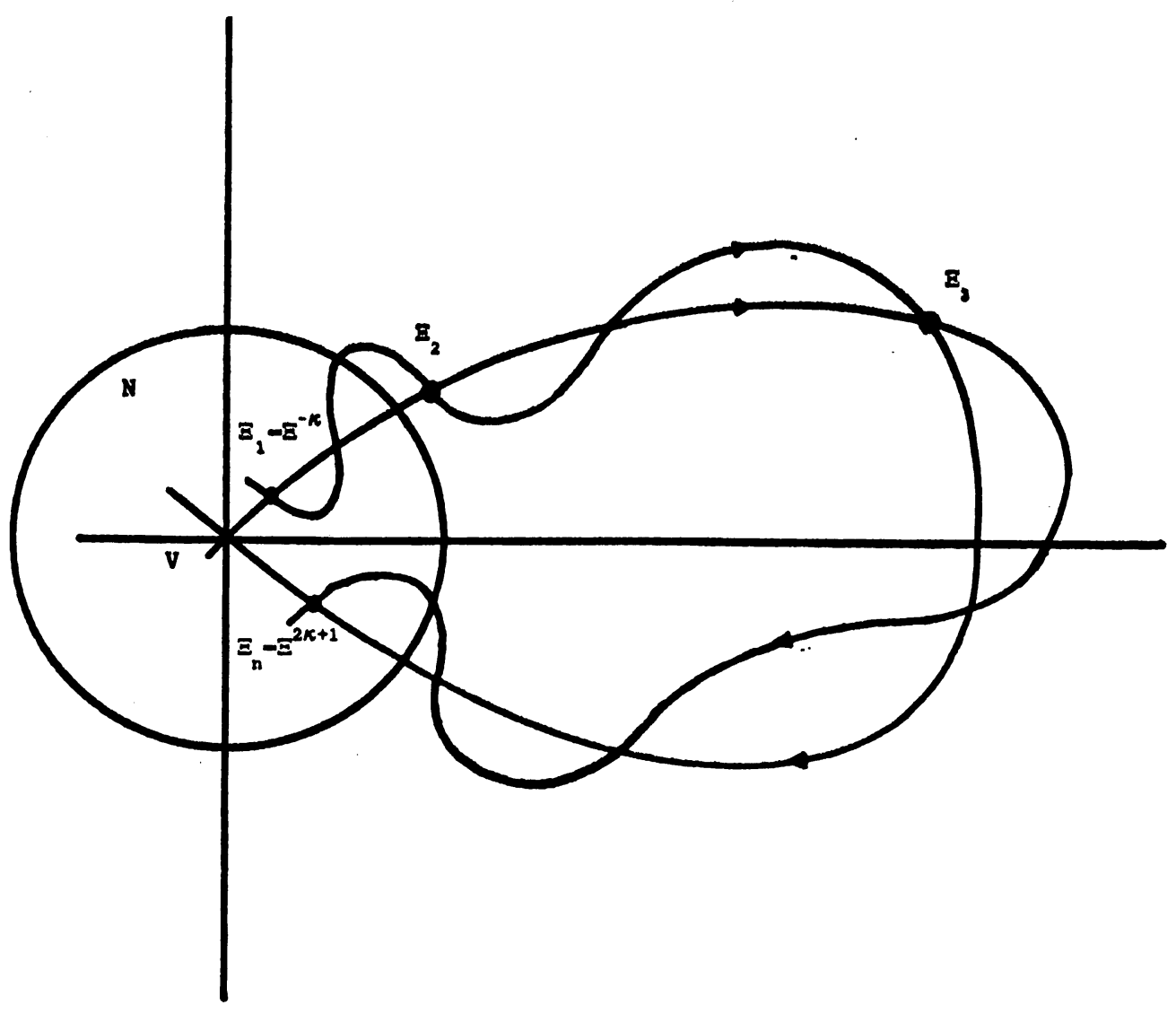

Figure 9: A portion of the hyperbolic set $\Lambda$

our starting point let it be denoted by $\Xi_{1}$ and, in general, let $\Xi_{i}=\Psi^{i-1}\left(\Xi_{1}\right)=\Xi^{-\kappa+i-1}$ for $1<i \leq n_{0}$ where $n_{0}=2 \kappa+1$. Also by the above for all $n \geq n_{0}, \Xi_{n}$ is in $N$. The Poincare map takes the set $\Xi_{1}$, which is in $N$, to the set $\Xi_{2}$, then to the set $\Xi_{3}$, etc. until it taken to $\Xi_{n}$, which is again back in $N$. See Figure $9 .^{8}$ (In the periodic case the Poincaré map is just a diffeomorphism of the plane and the sets $\Xi_{i}$ are just points as illustrated in Figure 9.) By the choice of the neighborhood $N$ of $V$ any jump within $N$ is allowed in an $\alpha$-pseudo orbit and in particular remaining at $\Xi_{n}$ is allowed.

Let $n \geq n_{0}$ be fixed. We will now describe the $\alpha$-pseudo orbits for (3.1) which are of particular interest here. As always an $\alpha$-pseudo orbit is a bisequence $\left\{\left(x_{i}, f_{i}\right)\right\}$ with $f_{i+1}=\eta\left(f_{i}\right)$ and $d\left(\Psi\left(x_{i}, f_{i}\right), x_{i+i}\right)<\alpha$, however we require more. In addition we ask that

$$
\left(x_{i}, f_{i}\right) \in \Xi_{r}, \quad \text { for some } r, 1 \leq r \leq n
$$

and for all $i$. Furthermore we require that if $\left(x_{i}, f_{i}\right) \in \Xi_{r}$ then one can have $\left(X_{i+1}, f_{i+1}\right) \in$ $\Xi_{s}$ only if $k_{r s}=1$, where $k_{r s}$ is the $r s$-term in the transition matrix $K$ given by (2.7).

\footnotetext{
${ }^{8}$ Compare Figures 5 and 9.
} 
Finally with the exception of the case where $\left(x_{i}, f_{i}\right),\left(x_{i+1}, f_{x+1}\right) \in \Xi_{n}$ we require that $x_{i+1}=\Psi\left(x_{i}, f_{i}\right)$.

We wish to write a code for these $\alpha$-pseudo orbits. In particular we shall parameterize them by $q$ where $q \in B_{n}(K)$, the Bernoulli space. For each $f \in Z$ and $1 \leq \ell \leq n$, where $n \geq n_{0}$, there is a unique point $\xi_{\ell}(f, \varepsilon)$ in $R^{2}$ with $\left(\xi_{\ell}(f, \varepsilon), f\right) \in \Xi_{\ell}$ by Theorem 2. Let $q \in B_{n}(K)$ and let $z_{i}=\eta^{i}(f)$ be an $\eta$-orbit. Then $\left\{\left(\xi_{q_{i}}\left(z_{i}, \varepsilon\right), z_{i}\right)\right\}$ is an $\alpha$-pseudo orbit. By the Skew Shadow Lemma there is a point $p \in R^{2}$ such that $\Psi^{i}(p, f)$ is $\beta$-shadow for this $\alpha$-pseudo orbit. Define a map $\Upsilon: B_{n}(K) \times Z \rightarrow R^{2} \times Z$ by $\Upsilon(q, f)=(p, f)$ where $p$ is defined by the process given above. $\Upsilon$ is continuous since it is defined by the shadowing lemma which in turn was proved by appling the implicit function theorem which insures continuous dependence of solutions.

To see that $\Upsilon$ is one-to-one let $\Upsilon(q, f)=(p, f)$ and $\Upsilon(\bar{q}, f)=(\bar{p}, f)$ where $q \neq \bar{q}$. The $\Upsilon$-orbits through $(p, f)$ and $(\bar{p}, f)$ are $\beta$-shadows of different pseudo-orbits and, since $\operatorname{dist}\left(\Xi^{0}, \Xi^{i}\right)>4 \beta$ for $i \neq 0$, these pseudo-orbits differ by at least $4 \beta$ in one entry, say the $k^{\text {th }}$. Since the actual orbits $\beta$-shadow these pseudo orbits $\operatorname{dist}\left(\Psi^{k}(p, f), \Psi^{k}(\bar{p}, f)\right)>2 \beta$ and these $\Psi$-orbits are different. Thus $\Upsilon$ is a continuous, one-to-one mapping of a compact Hausdorff space, and so it is a homeomorphism onto its image $\Omega_{n} \subset R^{2} \times Z$.

Let $(q, f) \in B_{n}(K) \times Z,(r, g)=(A(q), \eta(f)), \Upsilon(q, f)=(p, f), \Upsilon(r, g)=(s, g)$. By the above construction the $\Psi$-orbits through $\Psi(p, f)$ and $(s, g) \beta$-shadow each other. By uniqueness $\Psi^{i+1}(p, f)=\Psi^{i}(s, g)$ or

$$
\Psi \circ T(q, f)=\Upsilon \circ(A \times \eta)(q, f) .
$$

Consequently $\Psi \mid \Omega_{n}$ is equivalent to $A \times \eta$. In other words, the diagram

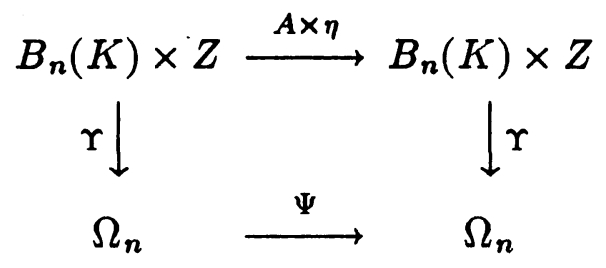

is commutative.

The set $\Omega_{n}$ is an invariant set for the Poincaré map $\Psi$. This generates an invariant set $\left[\Omega_{n}\right]$ in the skew product flow generated by (3.1). As a matter of fact $\left(\left[\Omega_{n}\right], \Pi\right)$ is the suspension of the discrete flow $\left(\Omega_{n}, \Psi\right)$ and $\Omega_{n}$ is a global cross section for $\left[\Omega_{n}\right]$. Since $\left(B_{n}(K) \times Z, A^{*} \eta\right)$ and $\left(\Omega_{n}, A \otimes \eta\right)$ are flow isomorphic, it follows that the continuous flows $\left(\mathcal{B}_{n}(K), \Psi\right)$ and $\left(\left[\Omega_{n}\right], \Pi\right)$ are flow isomorphic. We have thus proved the following result:

THEOREM 6. Let $H$ be a compact invariant set in $\mathcal{G}$ which is a proper Melnikov perturbation. Then there is an $\varepsilon_{0}>0$ such that for every $\varepsilon$ with $0<|\varepsilon|<\varepsilon_{0}$ there is an integer $n_{0}$ such that for every $n \geq n_{0}$ the skew product flow generated by (3.1) 
has an invariant set $\left[\Omega_{n}\right]$ which is flow isomorphic to the flow on the Bernoulli boundle $\mathcal{B}_{n}(K)=B_{n}(K) \otimes H$.

We can also prove the following result:

TheOREM 7. Let the hypotheses of Theorem 6 be satisfied and let $\varepsilon_{0}$ be given by Theorem 6. Then for every $\varepsilon, 0<|\varepsilon|<\varepsilon_{0}$ the skew product flow generated by (3.1) has an invariant set $\left[\Omega_{\infty}\right]$ which is flow isomorphic to the Bernuoulli bundle $\mathcal{B}_{\infty}=B_{\infty} \otimes H$, where $\left(B_{\infty}, A\right)$ is the full shift on a countable number of symbols.

Proof. We will just outline the proof here by showing how to modify the argument used in Theorem 5. In the proof of Theorem 5 we had constructed sets $\Xi_{1}, \Xi_{2}, \ldots, \Xi_{n_{0}}$, $\ldots, \Xi_{n}$ where $\Xi_{1} \subset N$ and $\Xi_{k} \subset N$ for $k \geq n_{0}$. Rather than stopping at $n$ one would continue and let $n \rightarrow \infty$. One then has a countable collection $\Xi_{1}, \Xi_{2}, \ldots$ where $\Xi_{k} \subset N$ for all $k \geq n_{0}$.

Now let $q=\ldots q_{-1} q_{0} . q_{1} q_{2} \ldots \in B_{\infty}$. We use $q$ to construct an $\alpha$-pseudo-orbit by the following rule:

$$
\begin{gathered}
\Xi_{1} \rightarrow \Xi_{2} \rightarrow \cdots \rightarrow \Xi_{n_{0}} \rightarrow \Xi_{n_{0}+1} \rightarrow \cdots \rightarrow \Xi_{n_{0}+q_{i}} \rightarrow \Xi_{1} \\
\rightarrow \Xi_{2} \rightarrow \cdots \rightarrow \Xi_{n_{0}} \rightarrow \Xi_{n_{0}+1} \rightarrow \cdots \rightarrow \Xi_{n_{0}+q_{i+1}} \rightarrow \Xi_{1}
\end{gathered}
$$

and so forth. In other words, each $q_{i}$ is used to fix the time that an $\alpha$-pseudo orbit remains in $N$ on each cycle.

The corresponding $\beta$-shadow then defines a compact invariant set $\Omega_{\infty}$ for the Poincaré map. The corresponding invariant set $\left[\Omega_{\infty}\right]$ for the skew product flow generated by (3.1) has the desired properties.

\section{An Illustrative Example}

We return to Duffing's equation but now with a different perturbation. In particular consider the partially coupled equation

$$
\begin{aligned}
& \dot{x}_{1}=x_{2} \\
& \dot{x}_{2}=x_{1}-x_{1}^{3}+\varepsilon f(\theta) \\
& \dot{\theta}=\omega+\varepsilon g(\theta)
\end{aligned}
$$

on $R^{2} \times T^{2}$ where $f: T^{2} \rightarrow R$ is continous, $g: T^{2} \rightarrow T^{2}$ is smooth and $\omega=\left(\omega_{1}, \omega_{2}\right)$ is constant with $\omega_{1} / \omega_{2}$ irrational. To be more specific take

$$
f(\theta)=f\left(\theta_{1}, \theta_{2}\right)=a_{1} \cos \theta_{1}+a_{2} \cos \theta_{2}
$$

where $a_{1}, a_{2}$ are nonzero. Let $\theta^{\varepsilon}(t)=\theta^{\varepsilon}\left(\theta_{0}, t\right)$ denote the solution of the $\theta$-equation with initial condition $\theta^{\varepsilon}(0)=\theta_{0}$. As shown in Section III.5, the Melnikov transform $M\left(f\left(\theta_{\tau}^{\varepsilon}\right)\right)$ 
has a simple zero set $Z^{0} \subset H=T^{2}$ at $\varepsilon=0$. Furthermore $Z^{0}$ is also a global cross section for the flow $\theta^{0}$ on $T^{2}$. Since $\theta^{\varepsilon}\left(\theta_{0}, t\right) \rightarrow \theta^{0}\left(\theta_{0}, t\right)$ as $\varepsilon \rightarrow 0$, uniformly for $t$ in compact sets in $R, Z^{0}$ is a global cross section for the flow $\theta^{\varepsilon}$ on $T^{2}$ for $\varepsilon$ small and nonzero. If $\theta_{0} \in Z^{0}$, then $M\left(f\left(\theta^{0}\left(\theta_{0}, \tau+\cdot\right)\right)\right)$ has a simple zero at $\tau=0$. By continuity for $\varepsilon$ small there is a $\hat{\theta}_{0}$ near $\theta_{0}$ such that $M\left(f\left(\theta^{\varepsilon}\left(\hat{\theta}_{0}, \tau+\cdot\right)\right)\right)$ has a simple zero at $\tau=0$. The collection $Z^{\varepsilon}$ of all such $\hat{\theta}_{0}$ forms another global cross section to the flow $\theta^{\varepsilon}$ on $T^{2}$.

Let $H_{\varepsilon}$ denote the collection of all functions in $\mathcal{G}$ of the form $t \rightarrow f\left(\theta^{\varepsilon}\left(\theta_{0}, t\right)\right)$ for $\theta_{0} \in T^{2} . H^{\varepsilon}$ is a compact invariant set in $\mathcal{G}$ and the translational flow on $H^{\varepsilon}$ is the (flow) homomorphic image of the flow $\theta^{\varepsilon}$ on $T^{2}$, i.e., the diagram

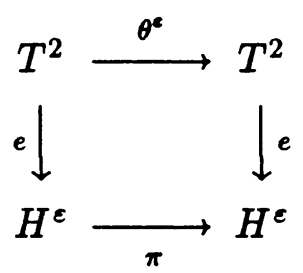

is communtative, where $e: T^{2} \rightarrow H^{\varepsilon}$ is defined by $e\left(\theta_{0}\right)(t)=f\left(\theta^{\varepsilon}\left(\theta_{0}, t\right)\right)$. Furthermore $e\left(Z^{\varepsilon}\right)$ is a global cross section to the translational flow on $H^{e}$.

Based on these considerations, equation (6.1) takes on the equivalent form

$$
\begin{aligned}
& \dot{x}_{1}=x_{2} \\
& \dot{x}_{2}=x_{1}-x_{1}^{3}+\varepsilon f(t)
\end{aligned}
$$

where $f \in H^{\varepsilon}$. The theory described in Theorems 5,6 and 7 applies to this problems, and we conclude that for $\varepsilon$ small and nonzero, and for $n \geq n_{0}$ equation (6.1) has a Bernoulli bundle flow $\mathcal{B}_{n}(K)=B_{n}(K) \otimes H^{\varepsilon}$ imbedded in the induced skew product flow on $R^{2} \times H^{\varepsilon}$.

\section{REFERENCES}

V. M. Alekseev (1976), Symbolic dynamics, 11th Math. School, Ed. Mitropolskii and Samoilenko, Kiev.

Z. ARTSTEIn (1977), Topological dynamics of ordinary differential equations and Kurzweil equations, J. Diff. Eqs., 23, pp. 224-243.

A. S. Besicovitch (1932), Almost Periodic Functions, Cambridge Univ. Press.

G. D. Birkн оғ (1932), Nouvelles recherches sur les systems dynamiques, Mem. Pont. Acad. Soc. Novi. Lyncaei, 1, pp. 85-216.

H. BонR (1925A), Zur Theorie der fastperiodischen Funktionen I, Acta Math., 45, pp. 29-127.

H. Boнr (1925B), Zur Theorie der fastperiodischen Funktionen II, Acta Math., 46, pp. 101-214.

H. BонR (1926), Zur Theorie der fastperiodischen Funktionen III, Acta Math., 47, pp. 237-281.

H. Bohr (1959), Almost Periodic Functions, Chelsea Publ., New York.

R. Bowen (1970), Equilibrium States and the Ergodic Theory of Anosov Diffeomorphisms, Lectures Notes in Mathematics, 470, Springer-Verlag, New York.

M. L. CARTwRight AND J. E. LitTlewood (1945), On non-linear differential equations of second order: I. The equation $\ddot{y}-k\left(1-y^{2}\right) \dot{y}+y=b \lambda k \cos (\lambda t+a), k$ large, J. London Math. Soc., 20, pp. 180-189.

S. N. Chow ANd J. K. Hale (1982), Methods of Bifurcation Theory, Springer-Verlag, New York. 
S. N. Chow, J. K. Hale and J. Mallet-Paret (1980), An example of bifurcation to homoclinic orbits, J. Diff. Eqs., 37, pp. 351-373.

C. C. Conley (1978), Isolated Invariant Sets and the Morse Index, CBMS Regional Conference, Amer. Math. Soc., Providence.

W. A. Coppel (1965), Stability and Asymptotic Behavior of Differential Equations, Heath, Boston.

W. A. Coppel (1978), Dichotomies in Stability Theory, Lect. Notes in Math., Springer-Verlag, New York.

C. Corduneanu (1968), Almost Periodic Functions, Interscience, New York.

I. P. Cornfeld, S. V. Fomin and Ya. G. Sinai (1982), Ergodic Theory, Springer-Verlag, New York.

R. Devaney (1986), An Introduction to Chaotic Dynamical Systems, Benjamin Commings, Menlo Park, California.

N. Ercolani, M. G. Forest and D. W. Mclaughlin (1987), Homoclinic orbits for the periodic sine-Gordon equation, preprint.

J. FaVArd (1933), Lecons sur les fonctions presque periodiques, Gauthier-Villars, Paris.

A. M. Fink (1974), Almost Periodic Differential Equations, Lect. Notes in Math., 377, Springer-Verlag, New York.

J. Guckenheimer and P. Holmes (1983), Nonlinear Oscillations, Dynamical Systems, and Bifurcations of Vector Fields, Springer-Verlag, New York.

J. K. HALE (1969), Ordinary Differential Equations, Wiley-Interscience, New York.

J. G. Hocking AND G. S. Young (1961), Topology, Addison-Wesley, New York.

P. Holmes (1980), Averaging and chaotic motion in forced oscillations, SIAM Appl. Math., 38, pp. 68-80.

G. IKegami (1969), On classification of dynamical systems with cross sections, Osaka Math. J., 6, pp. 419-433.

B. M. Levitan And V. V. Zhikov (1982), Almost Periodic Functions and Differential Equations, Cambridge Univ. Press., Cambridge.

L. Markus and K. R. Meyer (1980), Periodic orbits and solenoids in generic Hamiltonian systems, Amer. J. Math., 102, pp. 25-92.

J. E. Marsden (1984), Chaos in dynamical systems by the Poincaré-Melnikov-Arnold method, Proc. ARO Workshop on Dynamics, SIAM.

V. K. Melnikov (1963), On the stability of the center for time periodic perturbations, Trans. Moscow Math. Soc., 12, pp. 1-57.

K. R. Meyer and G. R. Sell (1987A), An analytic proof of the shadowing lemma, Funkcialaj Ekvacioj, 30, pp. 127-133.

K. R. Meyer and G. R. Sell (1987B), Homoclinic orbits and Bernoulli bundles in almost periodic systems, Oscillations, Bifurcatons and Chaos, CBMS Conference Proceedings, pp. 527-544.

K. R. Meyer and G. R. Sell (1987c), A model equation for describing chaos in the perturbed sine-Gordon equation.

R. K. Miller (1965), Almost periodic differential equations as dynamical systems with applications to the existence of a.p. solutions, J. Diff. Eqs., 1, pp. 337-345.

R. K. Miller ANd G. R. Sell (1970), Volterra integral equations and topological dynamics, Memoirs Amer. Math. Soc., 102.

M. MORSE (1921), A one-to-one representation of geodesics on a surface of negative curvature, Amer. J. Math., 43, pp. 33-51.

V. V. Nemyckil and V. V. Stepanov (1960), Qualitative Theory of Differential Equations, Princeton Univ., Princeton, N. J.

D. A. Neumann (1976), Dynamical systems with cross sections, Proc. Amer. Math. Soc., 58, pp. 339-344.

J. Palis and W. De Melo (1980), Geometric Theory of Dynamical Systems,, Springer-Verlag,, New York.

K. J. Palmer (1984), Exponential dichotomies and transversal homoclinic points, J. Diff. Eqs., 55, pp. 225-156.

H. Poincaré (1892), Les Méthodes Nouvelles de la Mécanique Céleste III, Gauthier-Villars, Paris.

H. G. Poinkhoff (1973), An analytic closing lemma, Proc. Midwest Dyn. Sys. Sem., Northwestern Univ. Press, Evanston, Ill, pp. 128-256. 
L. S. Pontryagin (1966), Topological Groups, 2nd ed., Gordon and Breach, New York.

R. J. SACKER AND G. R. SELl (1974), Existence of dichotomies and invariant splitting for linear differential systems I, J. Diff. Eqs., 15, pp. 429-458.

R. J. SACKer AND G. R. Sell (1977), Lifting properties in skew-product flows with applications to differential equations, Memoirs Amer. Math. Soc., 190.

J. SCHEURLe (1986), Chaotic solutions of systems with almost periodic forcing, preprint.

S. SchwabiK (1985), Generalized Differential Equations, Czechoslovakian Akad. Sci., Prague.

G. R. SELl (1967), Nonautonomous differential equations and topplogical dynamics I, II, Trans. Amer. Math. Soc., 127, pp. 241-262 and 263-283.

G. R. Sell (1971), Topological Dynamics and Ordinary Differential Equations, Van Nostrand, New York.

G. R. SELL (1978), The structure of a flow in the vicinity of an almost periodic motion, J. Diff. Eqs., 27(3), pp. 359-93.

YA. G. Sin AI (1973), Introduction to Ergodic Theory, English translation, Princeton Univ. Press, Princeton.

S. SMAle (1963), Diffeomorphisms with infinitly many periodic points, Differential and Combinatorial Topology, S. Chern (ed.), pp. 63-80, Princeton Univ. Press.

S. Wiggens (1986A), A generalization ot the method of Melnikov for detecting chaotic invariant sets, preprint.

S. WIGGENS (1986B), The orbit structure in the neighborhood of a transverse homoclinictorus, preprint. 